REPORTS ON MATHEMATICAL LOGIC

52 (2017), 101-132

doi:10.4467/20842589RM.17.006.7144

Mirjana ILIĆ

\title{
A NATURAL DEDUCTION AND ITS CORRESPONDING SEQUENT CALCULUS FOR POSITIVE CONTRACTION-LESS RELEVANT LOGIC
}

\begin{abstract}
A b s t r a c t. We give a normalizing system of natural deduction for positive contraction-less relevant logic $R W_{+}^{\circ}$. The specific characteristic of our calculus is that it has a simple translational relationship to a particular sequent calculus for $R W_{+}^{\circ}$, such that normal natural deduction derivations correspond to cut-free sequent calculus derivations and vice versa. By translations from natural deduction to sequent calculus derivations, and back, together with cut-elimination, we obtain an indirect proof of the normalization.
\end{abstract}

Received 7 April 2017

Key words and phrases: relevant logic, natural deduction. AMS subject classification: 03F05, 03F52. 


\section{Introduction}

Gentzen invented a calculus of natural deduction in [8]. His idea was to set up a formal system which comes as close as possible to actual reasoning and the result were natural deduction calculi NJ and NK, for intuitionistic and classical predicate logic.

In this paper we set up a natural deduction calculus for the logic $R W_{+}^{\circ}$, which is positive contraction-less relevant system $R$ (see [1], p. 341), with co-tenability $\circ$. Several natural deduction calculi are known for relevant logics, including $R W_{+}^{\circ}$, however, all of them, use a kind of relevance numerals in order to keep track of the use of hypotheses (see Anderson and Belnap [1], Dunn [6], [7], Brady [3], Urquhart [18], and Meyer and McRobbie [12]). This is needed to unable the inference of irrelevant formulae. Namely, the Gentzen rule $(\wedge I)$ :

$$
\frac{\alpha \quad \beta}{\alpha \wedge \beta}
$$

would license the inference of the irrelevant formula $\alpha \rightarrow(\beta \rightarrow \alpha)$, even when every application of the rule $(\rightarrow \mathrm{I})$ satisfies the requirement of discharging some assumption (as it is pointed out in Church [4]):

$$
\begin{gathered}
\frac{\alpha^{(1)} \beta^{(2)}}{\alpha \wedge \beta}(\wedge \mathrm{I}) \\
\frac{\alpha}{\beta \rightarrow \alpha}(\wedge \mathrm{E}) \\
\frac{\alpha \rightarrow(\beta \rightarrow \alpha)}{\alpha \rightarrow(2)}(\rightarrow \mathrm{I})^{(1)}
\end{gathered}
$$

Instead of the above rule $(\wedge I)$, Anderson and Belnap use in effect the following subscripted rule:

$$
\frac{\alpha_{a} \quad \beta_{a}}{\alpha \wedge \beta_{a}}
$$

(the subscript $a$ is the relevance numeral) in their Fitch-style natural deduction calculi for relevant logics in [1]. Each hypothesis in their derivation is subscripted with a distinct numeral which is then passed along each application of a rule, until the hypothesis is discharged. Then the subscript is dropped. A formula without subscript is a theorem, since it depends 
on no hypotheses. However, in Anderson and Belnap natural deduction calculi, subscripted introduction and elimination rules are insufficient for the proof of the distribution of conjunction over disjunction. In order to enable the inference of the formula $\alpha \wedge(\beta \vee \gamma) \rightarrow(\alpha \wedge \beta) \vee(\alpha \wedge \gamma)$, they add a special distribution rule. This rule is neither an introduction nor an elimination rule, therefore its presence in natural deduction calculi mismatches Gentzen's idea of natural deduction, where inference rules, i.e. introduction-elimination rules, provide not just a convenient format for proofs, but also the analysis (or meaning) of the connectives.

Dunn [6], [7] removes the need for the special distribution rule, by allowing hypotheses to be introduced in two different ways, 'relevantly' and 'irrelevantly' - 'relevantly' introduced hypotheses must all be used in the derivation, whereas only some (at least one) of the hypotheses 'irrelevantly' introduced, must be used. This is formalized by allowing several hypotheses, separated by commas, to be listed within a leaf of a derivation tree, but with a single relevance numeral attached to them (in fact, several formulae, separated by commas, are allowed to be listed on every line of a derivation tree). The comma is interpreted via $\wedge$ and the formulae on a line are interpreted as grouped into a set, to avoid the need for explicit structural rules corresponding to the commutation and idempotence of conjunction (although two explicit structural rules are still needed, one for the introduction and the other one for the elimination of the new structural connective).

By allowing several formulae to be listed on a line, Dunn introduced extensional (sometimes called additive) sets, into natural deduction. It should be mentioned that Dunn [5] and also Minc [13], discovered that two types of sequences, intensional and extensional, are needed in sequent calculi for relevant logics. Namely, to enable the inference of the distributive law in the absence of the structural rule of thinning, they used two kinds of structural connectives to build up antecedents of sequents: intensional, corresponding to $\circ$, and extensional ones, corresponding to $\wedge$, which must be allowed to be nested within one another. (We should note that Kron formulated sequent calculus for a contraction-less relevant logic based on intensional sequences only, where the inference of the distributive law is enabled by the explicit distribution rule; however, Kron's logic is different from $R W$, see [11].)

Brady sets up (normalizing Fitch-style) natural deduction calculi, for 
a wide range of relevant logics, including $R W_{+}^{\circ}$ in [3]. In order to remove the need for the special distribution rule, Brady allows, like Dunn, several formulae, separated by commas, to be listed on a line of a derivation tree. However, Brady's comma is interpreted as $\vee$, and it may be introduced only in the conclusion of the disjunction elimination rule (therefore Brady's derivations begin with single formulae, unlike Dunn's which may begin with extensional sets of formulae). Brady also uses relevance numerals, like Dunn, consisting of sets of natural numbers, possible empty, attached to each step of a proof, to denote the assumptions upon which the proof step depends.

Urquhart [18] also sets up a Fitch-style natural deduction calculus, where the distributive law follows from the introduction and elimination rules for conjunction and disjunction and where every line of a derivation consists of a single formula. However, Urquhart's system formalizes the positive semilattice system which is the extension of pure relevant implication.

Meyer and McRobbie [12] formulate natural deduction calculi of $R$ and a number of its neighbors, including $R W_{+}^{\circ}$, however with the explicit distribution rule. They allow only a single formula on a line of a proof, like Urquhart, but they use (intensional) multisets of active hypotheses, called tags, for keeping track of dependence relation.

In this paper we set up a natural deduction calculus $N R W_{+}^{\circ}$ for the logic $R W_{+}^{\circ}$, which is without explicit distribution rule and which is free from relevance numerals.

Some basic properties of our natural deduction calculus are:

1. The calculus is based on two kinds of multisets (lists without order) of formulae: intensional and extensional. Intensional multisets are interpreted via intensional conjunction $\circ$, whereas extensional ones are interpreted via extensional conjunction $\wedge$. We use two different punctuation marks to denote multisets: we use semicolons for intensional multisets and commas for extensional multisets. Greek capitals $\Gamma, \Pi, \Sigma, \Delta, \Gamma_{1}, \ldots$ denote intensional multisets of extensional multisets, of intensional multisets of, etc. of formulae, or extensional multisets of intensional multisets of extensional multisets of, etc. of formulae ('intensional' and 'extensional' must alternate). The formal definition of intensional and extensional multisets is given in Section 2. 
2. To start up the inference we use initial rules of the form:

$$
\frac{\alpha, \Gamma}{\alpha}
$$

where $\Gamma$ is possible empty. The standard rule of axiom $\alpha$, meaning that $\alpha$ can be derived from $\alpha$, i.e.

$$
\frac{\alpha}{\alpha}
$$

comes out as the special case of our initial rule.

Premises of initial rules are called assumption multisets. Formulae from assumption multisets are called assumptions. In our derivations, we shall use the standard notions of discharged (closed) and active (open) assumptions.

3. We discharge active assumptions, by the application of inference rules. The notation $\theta$, indicates that the assumption $\alpha$ is discharged or closed. Additionally, we use discharge labels, denoted by integers within parentheses, written next to mnemonic symbol for the rule applied and also next to the discharged assumptions. (Usually, discharge of an assumption in natural deduction systems, is denoted by square brackets, i.e., $[\alpha]$ denotes that the assumption $\alpha$ is discharged. However, in this paper square brackets will be used to denote the specific occurrence of a multiset within a multiset, e.g., we use $\Gamma[\alpha]$ to denote the specific occurrence of a formula $\alpha$ within a multiset $\Gamma$.) The specificity of our inferences is that open assumptions may move from one place in a derivation, to another, by the application of some inference rules. Moved assumptions are denoted by crossing out with thin lines - they are treated as being discharged at the former position, but open at the new one. Additionally we use move labels, indicated by integers (without parentheses, to distinguish them from discharge labels) written next to the moved assumptions and also next to mnemonic symbol for the rule applied. However, if the rule also discharges an assumption, then a moved and a discharge label are indicated by the same integer and then, only a discharge label appears next to mnemonic symbol for the rule applied. Given this, 
by a moved or a discharge label, we indicate the rule applied when an assumption is moved.

4. It is well-known that weakening corresponds to vacuous discharge of assumptions in natural deduction. Relevant logics are free from intensional weakening, but they are with extensional weakening, therefore one could expect some form of vacuous discharge of assumptions. However, in our calculus no vacuous discharge occurs (this is because of the form of our initial rules), i.e. every application of the rule $(\rightarrow \mathrm{I})$ satisfies the requirement of discharging some assumptions.

On the other hand, contraction corresponds to multiple discharge of assumptions in natural deduction. Our logic is free from intensional contraction, but it is with extensional contraction. Therefore, our calculus permits a form of multiple discharge of assumptions, i.e. in some cases it is possible to discharge several occurrences of a multiset at once.

5. We follow von Plato [16] and Negri [14], [15] in using general, instead of standard (special) elimination rules (as is well-known, special elimination rules are the special cases of the corresponding general elimination rules).

6. Our natural deduction calculus is without explicit distribution rule, as well as the calculi of Dunn and Brady. To illustrate the differences between those systems, we shall give, in the Appendix, the proofs of the formula $(\alpha \rightarrow(\beta \wedge \delta)) \wedge((\gamma \wedge \delta) \rightarrow \gamma) . \rightarrow .((\alpha \vee \gamma) \wedge(\alpha \vee \delta)) \rightarrow(\beta \vee \gamma)$ in all of them.

This paper is organized as follows. In Section 2, we give the sequent calculus $G R W_{+}^{\circ}$ (discussed in details in [10]), to which our natural deduction calculus has a simple translational relationship. In Section 3, we present the natural deduction system $N R W_{+}^{\circ}$. In Section 4, we define local translations between the sequent calculus $G R W_{+}^{\circ}$ and the natural deduction system $N R W_{+}^{\circ}$ and show that cut-free derivations are mapped into normal derivations, thus obtaining an indirect proof of normalization. 


\section{Sequent calculus $G R W_{+}^{\circ}$}

$G R W_{+}^{\circ}$ is presented in details in [10]. Here we only give some basic notions.

Let $\mathcal{L}$ be a language of the propositional calculus with $\rightarrow, \circ, \wedge$ and $\vee$. Formulae of $\mathcal{L}$ are defined inductively, as usual. We shall use $\alpha, \beta$, $\gamma, \varphi, \xi, \eta, \alpha_{1}, \ldots$ as schematic letters for formulae of $\mathcal{L}$.

A sequent is an expression of the form $\Gamma \vdash \gamma$, where $\Gamma$ is the antecedent and $\gamma$ is the succedent of a sequent. Antecedents are built up from intensional and extensional multisets which we define as follows:

\section{Definition.}

(i) Let $\alpha_{1}, \ldots, \alpha_{n}, n \geq 1$, be formulae of $\mathcal{L}$. Then $\left(\alpha_{1} ; \ldots ; \alpha_{n}\right)$ is the intensional, also called $i-$ multiset, and $\left(\alpha_{1}, \ldots, \alpha_{n}\right)$ is the extensional, also called $e-$ multiset. We note that a single formula is both, $i-$ and $e-$ multiset. A multiset which consists of a single formula is called atomic.

(ii) Let $\Gamma$ be an $i$-multiset of the form $\left(\Gamma_{1} ; \ldots ; \Gamma_{n}\right), n \geq 2$, and let $\alpha$ be a formula of $\mathcal{L}$. Then with $\Gamma ; \alpha$ or $\alpha ; \Gamma$ we denote the $i_{-}$ multiset $\left(\Gamma_{1} ; \ldots ; \Gamma_{n} ; \alpha\right)$ and with $\Gamma, \alpha$ or $\alpha, \Gamma$ we denote the $e-m u l t i s e t$ $\left(\left(\Gamma_{1} ; \ldots ; \Gamma_{n}\right), \alpha\right)$.

Let $\Gamma$ be an $e^{-m u l t i s e t}$ of the form $\left(\Gamma_{1}, \ldots, \Gamma_{n}\right), n \geq 2$, and let $\alpha$ be a formula of $\mathcal{L}$. Then with $\Gamma ; \alpha$ or $\alpha ; \Gamma$ we denote the $i_{-}$ multiset $\left(\left(\Gamma_{1}, \ldots, \Gamma_{n}\right) ; \alpha\right)$ and with $\Gamma, \alpha$ or $\alpha, \Gamma$ we denote the $e^{-}$ multiset $\left(\Gamma_{1}, \ldots, \Gamma_{n}, \alpha\right)$.

(iii) Let $\Gamma$ and $\Delta$ be two multisets. Then we have the following cases:

Let both $\Gamma$ and $\Delta$ be $i$-multisets, such that $\Gamma$ is of the form $\left(\Gamma_{1} ; \ldots ; \Gamma_{n}\right)$, $n \geq 2$, and $\Delta$ is of the form $\left(\Delta_{1} ; \ldots ; \Delta_{m}\right), m \geq 2$. Then with $\Gamma ; \Delta$ or $\Delta ; \Gamma$ we denote the $i-$ multiset $\left(\Gamma_{1} ; \ldots ; \Gamma_{n} ; \Delta_{1} ; \ldots ; \Delta_{m}\right)$ and with $\Gamma, \Delta$ or $\Delta, \Gamma$ we denote the $e-$ multiset $\left(\left(\Gamma_{1} ; \ldots ; \Gamma_{n}\right),\left(\Delta_{1} ; \ldots ; \Delta_{m}\right)\right)$.

Let one of $\Gamma$ and $\Delta$ be an $i-$ multiset, say $\Gamma$, and let it be of the form $\left(\Gamma_{1} ; \ldots ; \Gamma_{n}\right), n \geq 2$, and let the other one, $\Delta$, be an $e-m u l t i s e t$ of the form $\left(\Delta_{1}, \ldots, \Delta_{m}\right), m \geq 2$. Then with $\Gamma ; \Delta$ or $\Delta ; \Gamma$ we denote the $i$-multiset $\left(\Gamma_{1} ; \ldots ; \Gamma_{n} ;\left(\Delta_{1}, \ldots, \Delta_{m}\right)\right)$ and with $\Gamma, \Delta$ or $\Delta, \Gamma$ we denote the $e^{- \text {multiset }}\left(\left(\Gamma_{1} ; \ldots ; \Gamma_{n}\right), \Delta_{1}, \ldots, \Delta_{m}\right)$.

Let both $\Gamma$ and $\Delta$ be $e-$ multisets, such that $\Gamma$ is of the form $\left(\Gamma_{1}, \ldots, \Gamma_{n}\right)$, $n \geq 2$, and $\Delta$ is of the form $\left(\Delta_{1}, \ldots, \Delta_{m}\right), m \geq 2$. Then with $\Gamma ; \Delta$ or 
$\Delta ; \Gamma$ we denote the $i$-multiset $\left(\left(\Gamma_{1}, \ldots, \Gamma_{n}\right) ;\left(\Delta_{1}, \ldots, \Delta_{m}\right)\right)$ and with $\Gamma, \Delta$ or $\Delta, \Gamma$ we denote the $e-$ multiset $\left(\Gamma_{1}, \ldots, \Gamma_{n}, \Delta_{1}, \ldots, \Delta_{m}\right)$.

Note that intensional and extensional multisets are allowed to be nested within one another, but nested multisets of the same kind are not allowed.

As mentioned before, we use square brackets to denote a specific occurrence of a multiset within a multiset. Within a rule, square brackets localize, in upper sequents, an occurrence of a multiset within a multiset and, in lower sequents, the result of the replacement of an occurrence of a multiset by a multiset (with the understanding that substitution does not produce nested multisets of the same kind, e.g. a substitution of an intensional multiset for an item in an intensional multiset does not produce an intensional multiset with a nested element that is an intensional multiset).

$G R W_{+}^{\circ}$ has the following postulates:

Axiom:

$\alpha \vdash \alpha$

Structural rules ( $\Pi$ and $\Sigma$ are non-empty; all other multisets are possible empty):

$$
\begin{aligned}
& \text { extensional contraction: extensional weakening: } \\
& \frac{\Gamma[\Pi, \Pi] \vdash \gamma}{\Gamma[\Pi] \vdash \gamma}(\mathrm{WE}) \quad \frac{\Gamma[\Sigma] \vdash \gamma}{\Gamma[\Pi, \Sigma] \vdash \gamma}(\mathrm{KE}) \\
& \text { cut: } \\
& \frac{\Pi \vdash \varphi \quad \Gamma[\varphi] \vdash \gamma}{\Gamma[\Pi] \vdash \gamma} \text { (cut - i) } \quad \frac{\vdash \varphi \quad \Gamma[\varphi ; \Pi] \vdash \gamma}{\Gamma[\Pi] \vdash \gamma} \text { (cut - ii) } \frac{\vdash \varphi \quad \varphi \vdash \gamma}{\vdash \gamma} \text { (cut - iii) }
\end{aligned}
$$

where $\Gamma[\Pi]$ in (cut-i) is the result of replacing exactly one, displayed, occurrence of $\varphi$ in $\Gamma[\varphi]$ by $\Pi$; in (cut-ii), the single (displayed) occurrence of $\varphi$ in $\Gamma[\varphi ; \Pi]$ is replaced by an empty multiset; similarly for (cut-iii).

Operational rules:

$$
\begin{aligned}
& \frac{\Gamma_{1} \vdash \alpha \quad \Gamma_{2}[\beta] \vdash \gamma}{\Gamma_{2}\left[\Gamma_{1} ; \alpha \rightarrow \beta\right] \vdash \gamma}(\rightarrow 1) \\
& \frac{\alpha ; \Gamma \vdash \beta}{\Gamma \vdash \alpha \rightarrow \beta}(\rightarrow \mathrm{r}) \\
& \frac{\Gamma[\alpha ; \beta] \vdash \gamma}{\Gamma[\alpha \circ \beta] \vdash \gamma}(\circ 1) \\
& \frac{\Gamma_{1} \vdash \alpha \quad \Gamma_{2} \vdash \beta}{\Gamma_{1} ; \Gamma_{2} \vdash \alpha \circ \beta}(\circ \mathrm{r})
\end{aligned}
$$




$$
\begin{array}{lr}
\frac{\Gamma[\alpha] \vdash \gamma}{\Gamma[\alpha \wedge \beta] \vdash \gamma} \frac{\Gamma[\beta] \vdash \gamma}{\Gamma[\alpha \wedge \beta] \vdash \gamma}(\wedge \mathrm{l}) & \frac{\Gamma \vdash \alpha \quad \Gamma \vdash \beta}{\Gamma \vdash \alpha \wedge \beta}(\wedge \mathrm{r}) \\
\frac{\Gamma[\alpha] \vdash \gamma \quad \Gamma[\beta] \vdash \gamma}{\Gamma[\alpha \vee \beta] \vdash \gamma}(\vee 1) & \frac{\Gamma \vdash \alpha}{\Gamma \vdash \alpha \vee \beta} \frac{\Gamma \vdash \beta}{\Gamma \vdash \alpha \vee \beta}(\vee \mathrm{r})
\end{array}
$$

In [10] we prove:

Cut-Elimination Theorem. Every $G R W_{+}^{\circ}$-derivation can be transformed into a cut-free $G R W_{+}^{\circ}$-derivation with the same endsequent.

For our natural deduction derivations, with implicit extensional weakening rule, we shall need cut-free $G R W_{+}^{\circ}$-derivations, where the application of the rule $(\mathrm{KE})$ is controlled. We define a notion of a $\mathrm{KE}-$ normal proof, as follows.

Definition. A cut-free proof in $G R W_{+}^{\circ}$ is called KE-normal iff every application of (KE) in this proof is either the first rule of the proof, or it has only applications of (KE) above it, or it is the lower rule in the following contexts:

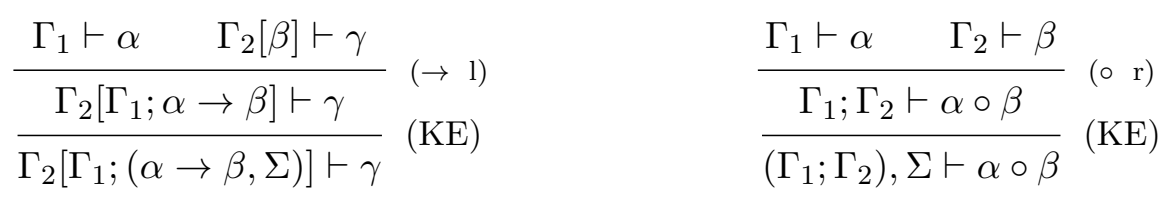

We prove the following lemma:

Lemma. For every cut-free $G R W_{+}^{\circ}$-derivation there is a cut-free $K E-$ normal $G R W_{+}^{\circ}$-derivation of the same endsequent.

Proof. Let $\pi$ be a cut-free $G R W_{+}^{\circ}$-derivation. We prove that $\pi$ can be transformed into a cut-free $\mathrm{KE}$-normal $G R W_{+}^{\circ}$-derivation of the same endsequent.

Let $S$ be the initial segment of $\pi$ which ends with the first from the top non-normal application of the rule (KE) and let (KE) be immediately preceded by the rule (r). Then we transform $S$, given on the left-hand side of $\mapsto$ into the derivation $S^{\prime}$, given on the right-hand side of $\mapsto$, as follows.

We have the following cases.

Let $(\mathrm{r})=(\mathrm{WE})$. Then we have: 


$$
\begin{array}{cc}
\pi_{1} & \pi_{1} \\
\frac{\Gamma[\Pi, \Pi] \vdash \gamma}{\Gamma[\Pi] \vdash \gamma}(\mathrm{WE}) & \mapsto \frac{\Gamma[\Pi, \Pi] \vdash \gamma}{\Gamma[\Pi, \Pi, \Sigma] \vdash \gamma}(\mathrm{KE}) \\
\frac{\Gamma[\Pi, \Sigma] \vdash \gamma}{\Gamma[\Pi, \Sigma] \vdash \gamma}(\mathrm{WE}) & \pi_{1} \\
\pi_{1} & \frac{\Gamma[\Pi, \Pi][\Theta] \vdash \gamma}{\Gamma[\Pi, \Pi][\Theta, \Sigma] \vdash \gamma}(\mathrm{KE}) \\
\frac{\Gamma[\Pi, \Pi][\Theta] \vdash \gamma}{\Gamma[\Pi][\Theta] \vdash \gamma}(\mathrm{WE}) & \mapsto \mathrm{KE}) \\
\frac{\Gamma[\Pi][\Theta, \Sigma] \vdash \gamma}{\pi_{1}} & \frac{\Gamma[\Pi[\Theta, \Sigma] \vdash \gamma}{\Gamma[\Pi[\Theta], \Pi[\Theta]] \vdash \gamma} \\
\frac{\Gamma[\Pi[\Theta], \Pi[\Theta]] \vdash \gamma}{\Gamma[\Pi[\Theta]] \vdash \gamma}(\mathrm{WE}) & \mapsto \frac{\pi_{1}}{\Gamma[\Pi[\Theta, \Sigma], \Pi[\Theta, \Sigma]] \vdash \gamma}(\mathrm{KE}) \\
\frac{\Gamma[\mathrm{KE})}{\Gamma[\Pi[\Theta, \Sigma]] \vdash \gamma}(\mathrm{KE})
\end{array}
$$

Let $(\mathrm{r})=(\rightarrow \mathrm{l})$. Then we have:

$$
\begin{array}{ccc}
\pi_{1} & \pi_{2} & \pi_{2} \\
\frac{\Gamma_{1} \vdash \alpha}{\Gamma_{2}\left[\Gamma_{1} ; \alpha \rightarrow \beta\right] \vdash \gamma} & \Gamma_{2}[\beta] \vdash \gamma \\
\frac{\Gamma_{2}\left[\Gamma_{1} ; \alpha \rightarrow \beta\right], \Sigma \vdash \gamma}{(\mathrm{KE})} & & \Gamma_{2}[\beta] \vdash \gamma \\
\left(\Gamma_{1} \vdash \alpha\right. & \Gamma_{2}[\beta], \Sigma \vdash \gamma \\
\Gamma_{2}\left[\Gamma_{1} ; \alpha \rightarrow \beta\right], \Sigma \vdash \gamma
\end{array}(\rightarrow 1)
$$

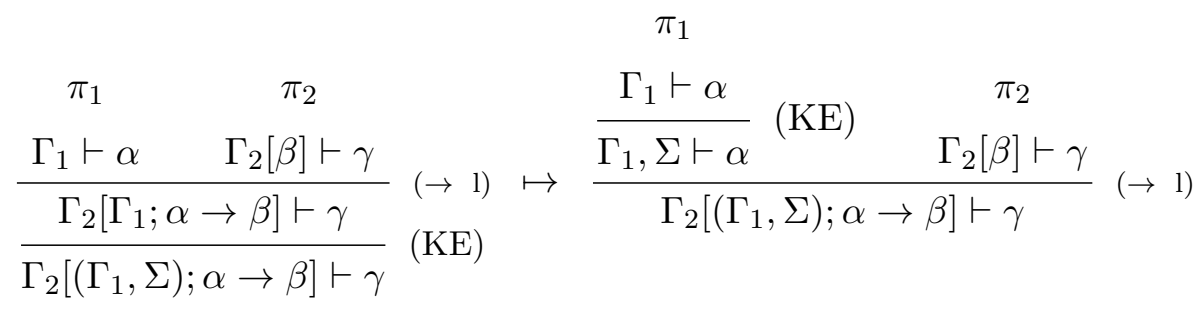

$$
\begin{aligned}
& \pi_{2}
\end{aligned}
$$

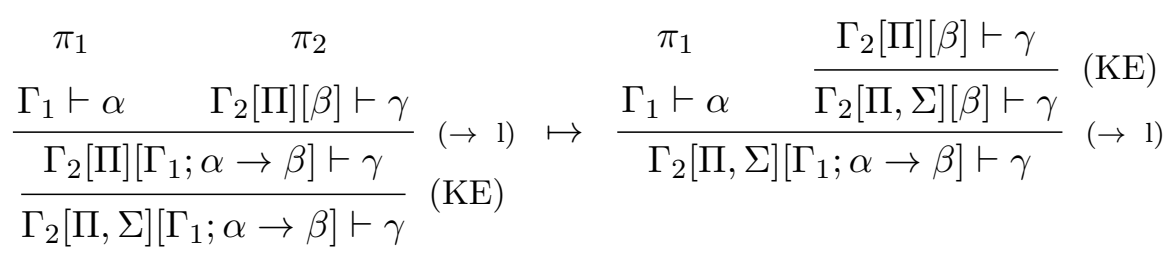

We note that $(\mathrm{KE})$ cannot be pushed above $(\rightarrow \mathrm{l})$ in: 


$$
\begin{array}{cc}
\pi_{1} & \pi_{2} \\
\frac{\Gamma_{1} \vdash \alpha \quad \Gamma_{2}[\beta] \vdash \gamma}{\Gamma_{2}\left[\Gamma_{1} ; \alpha \rightarrow \beta\right] \vdash \gamma} & (\rightarrow 1) \\
\Gamma_{2}\left[\Gamma_{1} ;(\alpha \rightarrow \beta, \Sigma)\right] \vdash \gamma & (\mathrm{KE})
\end{array}
$$

but this is the (KE)-normal proof.

Let $(r)=(\rightarrow r)$. Then we have:

$$
\begin{aligned}
& \pi_{1} \\
& \frac{\pi_{1}}{\frac{\alpha ; \Gamma \vdash \beta}{\Gamma \vdash \alpha \rightarrow \beta}} \underset{(\mathrm{KE})}{\Gamma \vdash \alpha \rightarrow \beta}(\mathrm{\textrm {K }}) \quad \mapsto \frac{\frac{\alpha ; \Gamma \vdash \beta}{\Gamma ;(\Gamma, \Sigma) \vdash \beta}}{(\mathrm{KE})}(\rightarrow \mathrm{r})
\end{aligned}
$$

Let $(\mathrm{r})=(\mathrm{\circ}$ l). Then we have:

$$
\begin{aligned}
& \pi_{1} \\
& \frac{\pi_{1}}{\frac{\Gamma[\alpha ; \beta] \vdash \gamma}{\Gamma[\alpha \circ \beta] \vdash \gamma}}(\circ \quad \text { r }) \mapsto \frac{\Gamma[\alpha ; \beta] \vdash \gamma}{\Gamma[(\alpha ; \beta), \Sigma] \vdash \gamma}(\mathrm{KE})
\end{aligned}
$$

$\pi_{1}$

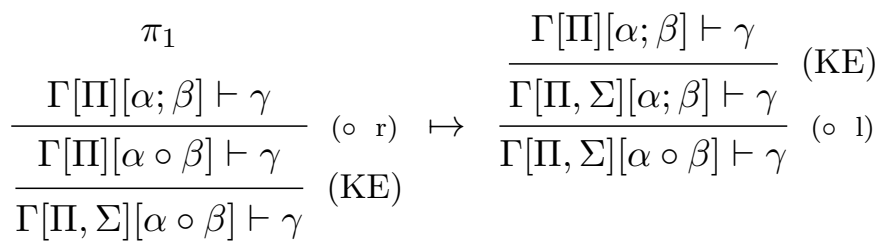

Let $(\mathrm{r})=(\mathrm{o} r)$. Then we have:

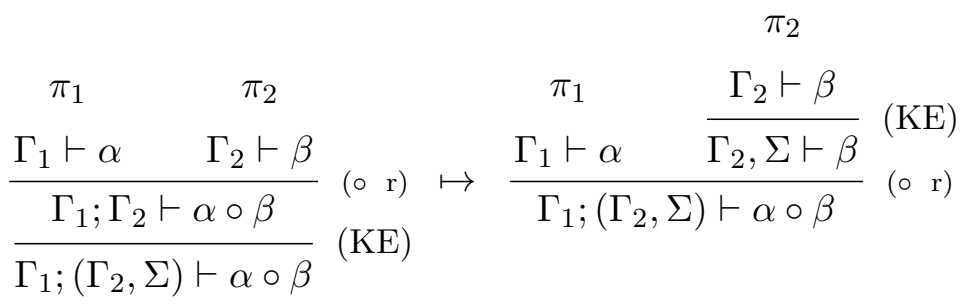

We can proceed similarly when the endsequent of $S$ is $\left(\Gamma_{1}, \Sigma\right) ; \Gamma_{2} \vdash \alpha \circ \beta$ 
and $\Sigma$ is the principal multiset of (KE). The rule (KE) cannot be pushed above (o r) in

$$
\begin{array}{cc}
\pi_{1} & \pi_{2} \\
\frac{\Gamma_{1} \vdash \alpha \quad \Gamma_{2} \vdash \beta}{\Gamma_{1} ; \Gamma_{2} \vdash \alpha \circ \beta} & (\circ \mathrm{r}) \\
\left(\Gamma_{1} ; \Gamma_{2}\right), \Sigma \vdash \alpha \circ \beta & (\mathrm{KE})
\end{array}
$$

but this is the (KE)-normal proof.

The transformations of $S$ can be obtained analogously when (r) is one of the rules $(\wedge \mathrm{l}),(\wedge \mathrm{r}),(\vee \mathrm{l})$ or $(\vee \mathrm{r})$. We continue to transform the proof, until we obtain a proof, where all applications of the rule (KE) are normal.

\section{Natural deduction calculus $N R W_{+}^{\circ}$}

We present the natural deduction system $N R W_{+}^{\circ}$ for positive contractionless relevant logic $R W_{+}^{\circ}$. Sometimes it is easier to understand if we call our logic intuitionistic linear logic without modalities, without constants 1 , $\top, 0, \perp$ and with distribution of conjunction over disjunction (the formula $\alpha \wedge(\beta \vee \gamma) \rightarrow(\alpha \wedge \beta) \vee(\alpha \wedge \gamma)$ is provable in $\left.R W_{+}^{\circ}\right)$. It should be mentioned that Sara Negri formulated the natural deduction system N-ILL, for intuitionistic linear logic, in [15], however, our system is, due to the presence of the distribution law, significantly different from N-ILL.

$N R W_{+}^{\circ}$ has the following postulates:

The initial rule (axiom) is of the form:

$$
\frac{\alpha, \Gamma}{\alpha}
$$

where $\Gamma$ is possible empty. 
Operational rules:

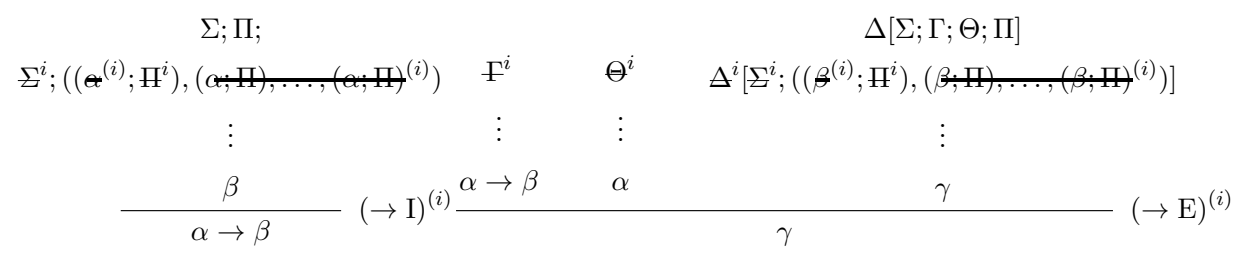

With $(\alpha ; \Pi),(\alpha ; \Pi), \ldots,(\alpha ; \Pi)$ we denote an extensional multiset where the multiset $\alpha ; \Gamma$ occurs at least once and where $\Pi$ may be empty. Similarly for $(\beta ; \Pi),(\beta ; \Pi), \ldots,(\beta ; \Pi)$.

The extensional contraction is implicit in our system, meaning that it is built in the inference rules. In the rule $(\rightarrow \mathrm{I})$, we implicitly apply the extensional contraction, by substituting the multiset $(\alpha ; \Pi),(\alpha ; \Pi), \ldots,(\alpha ; \Pi)$ with the multiset $\alpha$; $\Pi$, i.e. by discharging all emphasized occurrences of $(\alpha ; \Pi)$, except one (if there is more than one occurrence of the multiset $\alpha ; \Gamma$ within $(\alpha ; \Pi),(\alpha ; \Pi), \ldots,(\alpha ; \Pi))$. Then we introduce the implication $\rightarrow$, i.e. we derive the formula $\alpha \rightarrow \beta$ and discharge the formula $\alpha$ from the assumption multiset $\Sigma ; \alpha ; \Pi$, taking $\Sigma$ together with $\Pi$ instead; namely, in lieu of $\alpha$ we place the intensional multiset $\Sigma ; \Pi$, and we write it above the crossed out multiset $\Sigma ;((\alpha ; \Pi),(\alpha ; \Pi), \ldots,(\alpha ; \Pi))$. The discharged assumption $\alpha$, as well as the discharged multiset $(\alpha ; \Pi),(\alpha ; \Pi), \ldots,(\alpha ; \Pi)$, are crossed out by thicker lines than $\Sigma$ and $\Pi$ - the later assumption multisets are still open, however not at the starting position. The multiset $\Sigma ; \Pi$ is the multiset of moved open assumptions.

Similarly, in the rule $(\rightarrow \mathrm{E})$, we first substitute the multiset $(\beta ; \Pi),(\beta ; \Pi)$, $\ldots,(\beta ; \Pi)$ with the multiset $\beta ; \Pi$ and then we eliminate the implication $\rightarrow$ : we discharge the formula $\beta$ from the multiset $\Delta[\Sigma ; \beta ; \Pi]$ and we put the multiset of open assumptions $\Gamma ; \Theta$ in its place. The multiset of moved open assumptions $\Delta[\Sigma ; \Gamma ; \Theta ; \Pi]$ is written above the crossed out multiset $\Delta[\Sigma ;((\beta ; \Pi),(\beta ; \Pi), \ldots,(\beta ; \Pi))]$.

In both rules, the discharge label $(i)$ is new in the derivation so far constructed. 
The following restrictions are needed, when the rule $(\rightarrow \mathrm{E})$ is applied: if both $\Gamma$ and $\Theta$ are empty, then either at least one of $\Pi$ or $\Sigma$ is non-empty, or $\Delta[\Sigma ;((\beta ; \Pi),(\beta ; \Pi), \ldots,(\beta ; \Pi))]=\beta, \beta, \ldots, \beta$.

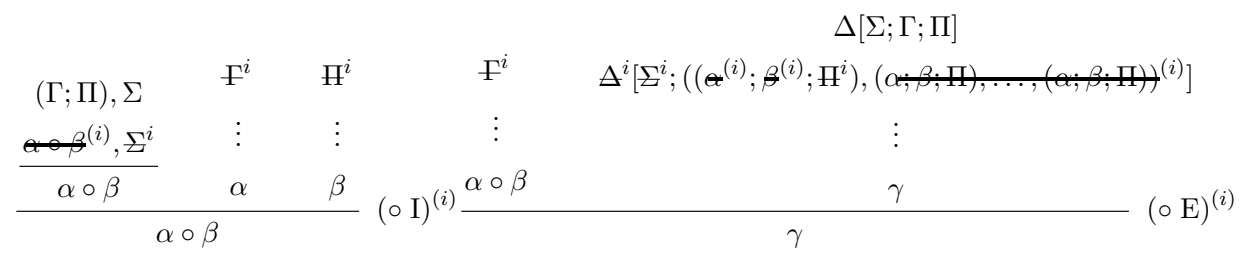

The following restrictions are needed: in the rule (o I), either $\Sigma$ is empty, or at least one of $\Gamma$ or $\Pi$ is non-empty; in the rule $(\circ \mathrm{E})$, if $\Gamma$ is empty, then either at least one of $\Pi$ or $\Sigma$ is non-empty, or

$$
\Delta[\Sigma ;((\alpha ; \beta ; \Pi),(\alpha ; \beta ; \Pi), \ldots,(\alpha ; \beta ; \Pi))]=(\alpha ; \beta),(\alpha ; \beta), \ldots,(\alpha ; \beta){ }^{1}
$$

\footnotetext{
${ }^{1}$ We note the unusual form of the rule (० I). Really, the usual formulation of this rule would be:

$$
\begin{array}{cc}
\Gamma & \Pi \\
\vdots & \vdots \\
\frac{\alpha}{\alpha} & \beta
\end{array}
$$
}

see e.g. [15]. We use different form of this rule to enable a translation of the following sequent derivation:

$$
\begin{array}{cc}
\vdots & \vdots \\
\frac{\Gamma_{1} \vdash \alpha \quad \Gamma_{2} \vdash \beta}{\frac{\Gamma_{1} ; \Gamma_{2} \vdash \alpha \circ \beta}{\left(\Gamma_{1} ; \Gamma_{2}\right), \Sigma \vdash \alpha \circ \beta}}(\mathrm{KE})
\end{array}
$$

into a natural deduction derivation with implicit weakening (see below). 


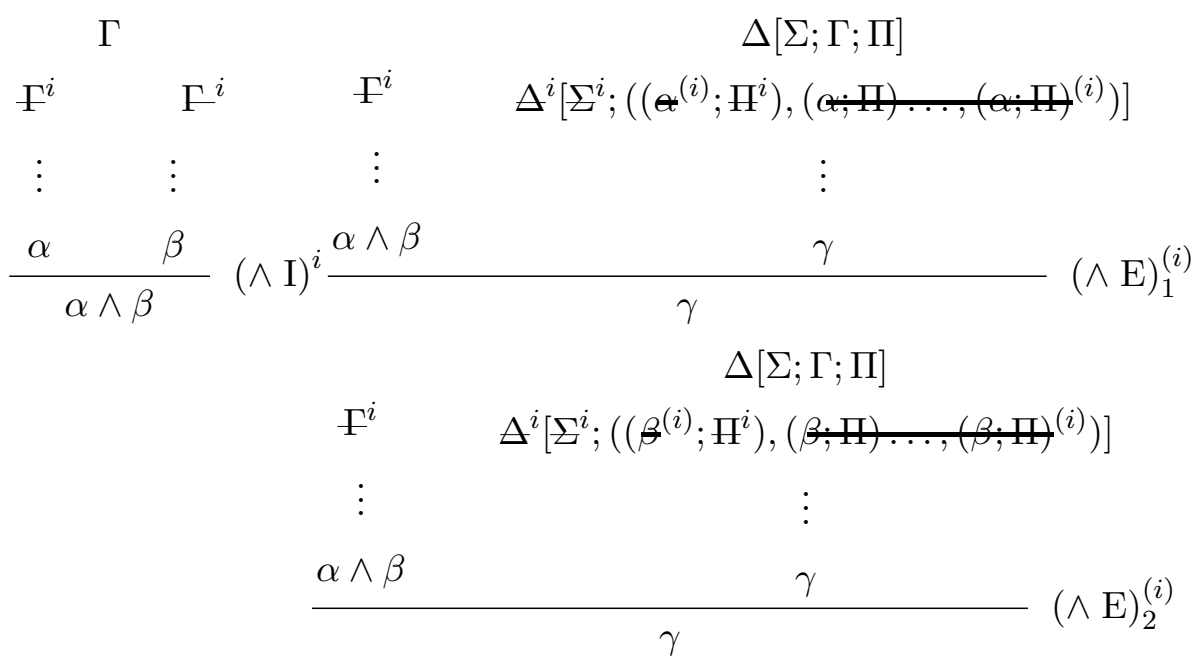

The following restrictions are needed: in the rule $(\wedge \mathrm{E})_{1}$, if $\Gamma$ is empty, then either at least one of $\Pi$ or $\Sigma$ is non-empty, or

$$
\Delta[\Sigma ;((\alpha ; \Pi),(\alpha ; \Pi), \ldots,(\alpha ; \Pi))]=\alpha, \alpha, \ldots, \alpha .
$$

Similarly for $(\wedge \mathrm{E})_{2}$.

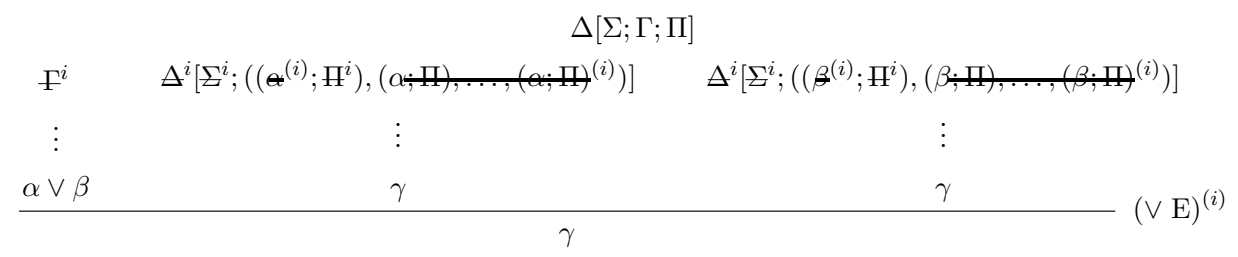

The following restrictions are needed: in the rule $(\vee E)$, if $\Gamma$ is empty, then either at least one of $\Pi$ or $\Sigma$ is non-empty, or we have that both $\Delta[\Sigma ;((\alpha ; \Pi),(\alpha ; \Pi), \ldots,(\alpha ; \Pi))]=\alpha, \alpha, \ldots, \alpha$ and $\Delta[\Sigma ;((\beta ; \Pi),(\beta ; \Pi), \ldots,(\beta ; \Pi))]=\beta, \beta, \ldots, \beta$.

In our elimination rules, we differentiate two types of premises: major and minor. The major premise is the premise whose principal connective is the connective of the rule in question, whereas all other premises are minor. The major premise appears on the left hand side of elimination rules.

A formula $\alpha$ is a theorem of $R W_{+}^{\circ}$ iff it depends on no hypotheses, i.e. iff there is an $N R W_{+}^{\circ}$-derivation, with $\alpha$ at the bottom, where all assumptions are discharged. 


\section{Translations}

We define translations from sequent calculus to natural deduction and vice versa. Our translations are defined bottom-up, i.e. we start with the last step of a derivation, working upwards step by step, until we reach axioms.

First we give a translation of a cut-free $\mathrm{KE}$-normal $G R W_{+}^{\circ}$-derivation $\pi$, of a sequent $\vdash \gamma$, to natural deduction. The last applied rule in $\pi$ can be one of: $(\rightarrow r),(\circ, r),(\wedge r)$ or $(\vee r)$. Then we have the following cases:

$$
\begin{aligned}
& \pi_{1} \quad \pi_{1} \\
& \frac{\alpha \vdash \beta}{\vdash \alpha \rightarrow \beta}(\rightarrow \mathrm{r}) \mapsto \frac{\mathrm{t}^{(i)} \vdash \beta}{\alpha \rightarrow \beta}(\rightarrow \mathrm{I})^{(i)} \\
& \begin{array}{llll}
\pi_{1} & \pi_{2} & \pi_{1} & \pi_{2}
\end{array} \\
& \frac{\vdash \alpha \quad \vdash \beta}{\vdash \alpha \circ \beta}(\circ \mathrm{r}) \mapsto \frac{\vdash \alpha \vdash \beta}{\alpha \circ \beta} \text { (० I) } \\
& \begin{array}{llll}
\pi_{1} & \pi_{2} & \pi_{1} & \pi_{2}
\end{array} \\
& \frac{\vdash \alpha \quad \vdash \beta}{\vdash \alpha \wedge \beta}(\wedge \mathrm{r}) \mapsto \frac{\vdash \alpha \quad \vdash \beta}{\alpha \wedge \beta}(\wedge \mathrm{I}) \\
& \begin{array}{cccc}
\pi & \pi & \pi & \pi \\
\frac{\vdash \alpha}{\vdash \alpha \vee \beta}(\vee \mathrm{r}) \mapsto \frac{\vdash \alpha}{\alpha \vee \beta}(\vee \mathrm{I}) & \frac{\vdash \beta}{\vdash \alpha \vee \beta}(\vee \mathrm{r}) \mapsto \frac{\vdash \beta}{\alpha \vee \beta}
\end{array}
\end{aligned}
$$

Every translation on the right-hand side of $\mapsto$ consists of the sequent calculus derivation(s) in the upper part, the rule in natural deduction notation and the conclusion of that rule in the lower part. The sequent calculus derivation(s) in the upper part will be called the sequent calculus segments of the translation, and they are the same as the corresponding sequent calculus derivations given on the left-hand side of $\mapsto$. Those derivations will be translated in the further steps of the translation.

The formula $\alpha$, in the sequent calculus segment of the first translation above, is assigned with the discharge label $(i)$ : this formula will be discharged by the application of the rule $(\rightarrow$ I). We cross out this formula, but we treat it in the same way as other formulae in subsequent translations.

Let $\operatorname{Tr}$ be the translation so far constructed and let one of its sequent calculus segments be the segment $S$, which is given on the left-hand side 
of $\mapsto$ below. If the last rule in $S$ is an operational rule, then we have:

$$
\frac{\alpha ; \Gamma \vdash \beta}{\mp \vdash \alpha \rightarrow \beta}(\rightarrow \mathrm{r}) \mapsto \frac{\pi}{\alpha \rightarrow \beta}(\rightarrow \mathrm{I})^{(i)}
$$

provided $(i)$ is new discharge label in the translation so far constructed. With $\mp$ we denote the multiset $\Gamma$ where every formula is with discharge label (although discharge labels are not visible). Furthermore, every formula from $\mp$ of the sequent $\mp \vdash \alpha \rightarrow \beta$ on the left hand side of $\mapsto$ has the same discharge label as its corresponding formula from $\mp$ of the sequent $\mathrm{A}^{(i)} ; \mp \vdash \beta$ on the right hand side of $\mapsto$. As mentioned before, crossed out formulae are treated in the same way as other formulae in subsequent translations.

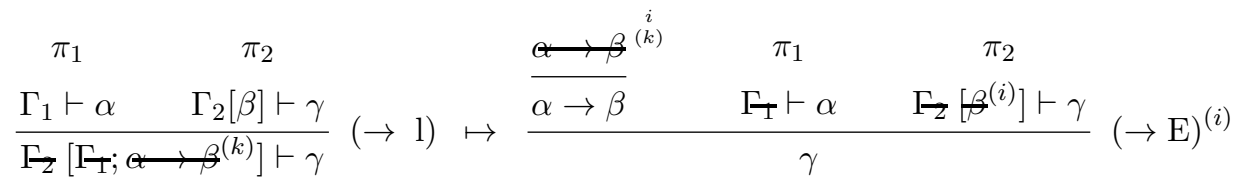

Superscripts $\underset{(k)}{i}$ indicate that the formula $\alpha \rightarrow \beta$ has the discharge label $(k)$ and moved label $i$.

$$
\begin{aligned}
& \begin{array}{lllll}
\pi_{1} & \pi_{2} & \beta^{(i)} & \pi_{1} & \pi_{2}
\end{array} \\
& \frac{\Gamma_{1} \vdash \alpha \quad \Gamma_{2} \vdash \beta}{\Gamma_{\mathbf{T}} ; \mathrm{F}_{\boldsymbol{Z}} \vdash \alpha \circ \beta}(\circ \mathrm{r}) \mapsto \frac{\overline{\alpha \circ \beta} \quad \mathrm{F}_{\mathbf{I}} \vdash \alpha \quad \mathrm{F}_{\boldsymbol{Z}} \vdash \beta}{\alpha \circ \beta}(\circ \mathrm{I})^{(i)}
\end{aligned}
$$

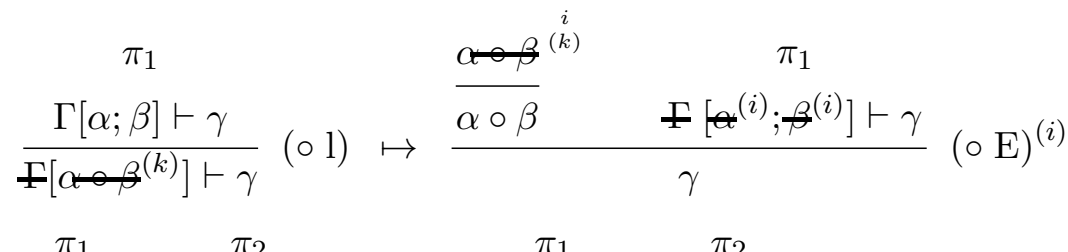

$$
\begin{aligned}
& \frac{\Gamma \vdash \alpha \quad \Gamma \vdash \beta}{\mp \vdash \alpha \wedge \beta}(\wedge \mathrm{r}) \mapsto \frac{\mp \vdash \alpha \quad \mp \vdash \beta}{\alpha \wedge \beta}(\wedge \mathrm{I})
\end{aligned}
$$

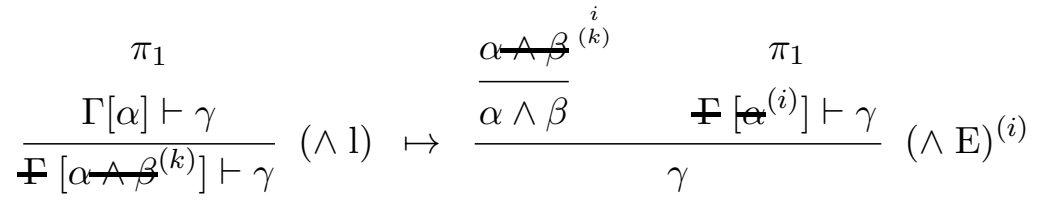




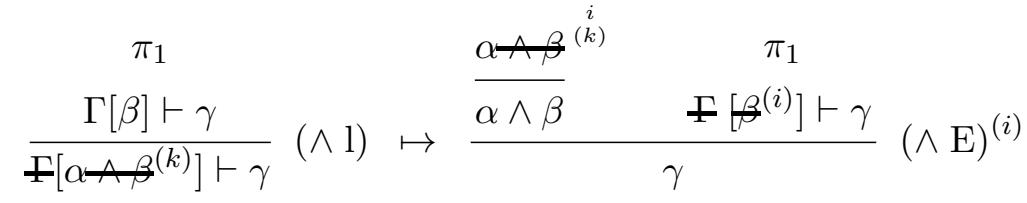

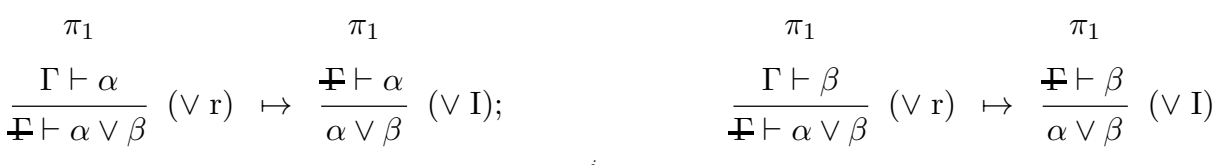

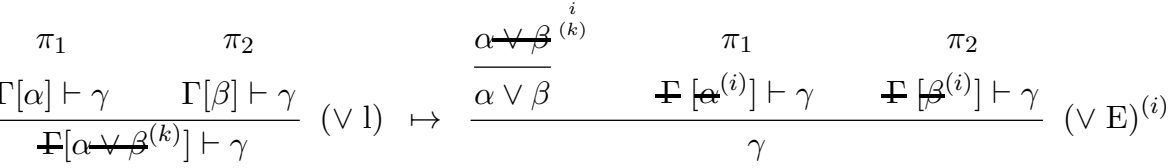

If the last rule in $S$ is structural, then we have:

$\pi_{1}$

$$
\frac{\Gamma[\Pi, \Pi] \vdash \gamma}{\mp[\Pi] \vdash \gamma}(\mathrm{WE}) \mapsto \begin{gathered}
\pi_{1} \\
\mathrm{~F}[\#, \Pi] \vdash \gamma
\end{gathered}
$$

(We duplicate the multiset of formulae $\Pi$, together with their discharge labels.)

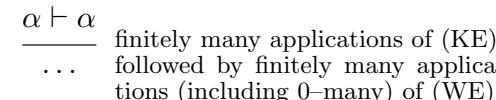

$$
\begin{aligned}
& \overline{\mathrm{e}^{(k)}, \boldsymbol{F} \vdash \alpha} \\
& \mapsto \frac{\mathrm{e}^{(k)}, \mathbf{F}}{\alpha} \\
& \pi_{1} \quad \pi_{2}
\end{aligned}
$$

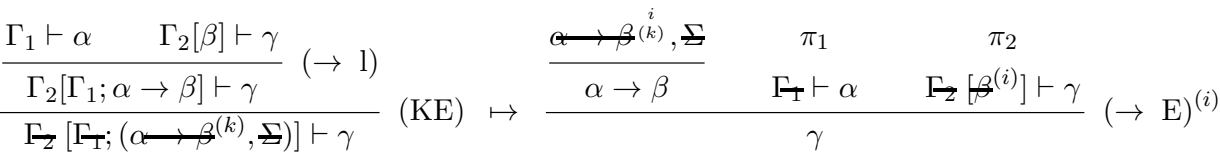

$$
\begin{aligned}
& \pi_{1} \quad \pi_{2}
\end{aligned}
$$

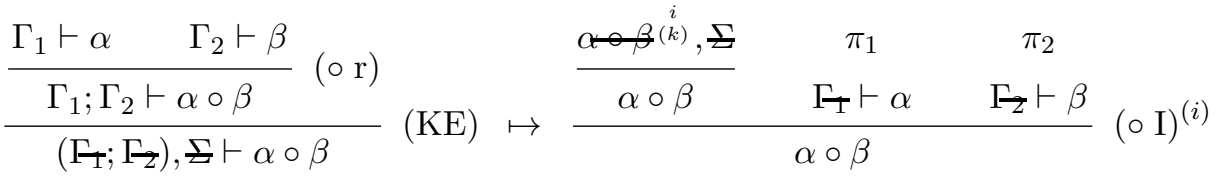


The translation of axioms is given as follows:

$$
\Theta^{(k)} \vdash \alpha \mapsto \frac{\boldsymbol{t}^{(k)}}{\alpha} \text {. }
$$

Our translation produces natural deduction derivations with discharges fully formalized. On the other hand, multisets of moved assumptions are missing, and therefore those derivations are not, strictly speaking, syntactically correct. To obtain syntactically correct derivations, we have to add them, which can easily be done, starting from the top (see the example in the Appendix, for a better understanding of how it should be done). The presence of multisets of moved assumptions in natural deduction derivations is crucial for the translation of those derivations into the sequent calculus derivation, as we shall see below.

In $N R W_{+}^{\circ}$, instead of standard (special) elimination rules, we use general elimination rules, for the reasons briefly explained in [16], [14] and [15]. Consequently, we can adopt the following definition of the normal derivation:

Definition. An $N R W_{+}^{\circ}$-derivation is normal when all major premises of elimination rules are conclusions of initial rules.

We note that in the above translations, all major premises of the elimination rules are conclusions of the initial rules, therefore our translations are normal natural deduction derivations. Thus we have proved:

Theorem 1. If $\pi$ is a cut-free $K E-$-normal $G R W_{+}^{\circ}$-derivation of a sequent $\vdash \gamma$, then its translation is a normal $N R W_{+}^{\circ}$-derivation of $\gamma$ from the empty multiset of open assumptions.

Now we give a translation of a natural deduction derivation of a formula $\gamma$ from a multiet of open assumptions $\Gamma$, to sequent calculus. Translation is defined inductively, according to the last rule used.

Multisets of open assumptions $\Pi, \Sigma, \Gamma$ and $\Theta$, which appear in our natural deduction derivations, are possible empty. The initial multisets in natural deduction derivations are either upper multisets of initial rules, or multisets of moved assumptions. 
If the last rule is $(\rightarrow \mathrm{I})$, then we have the following cases:

$\Pi$

$$
\begin{array}{cc}
\Pi^{i} ; \boldsymbol{\Theta}^{(i)} & \Pi ; \alpha \\
\vdots & \vdots \\
\frac{\beta}{\alpha \rightarrow \beta}(\rightarrow \mathrm{I})^{(i)} \mapsto \frac{\beta}{\Pi \vdash \alpha \rightarrow \beta}(\rightarrow \mathrm{r})
\end{array}
$$

Note that the formula $\alpha$, which is discharged on the left hand side of $\mapsto \quad$ is an open assumption on the right-hand side of $\mapsto$.

$$
\begin{aligned}
& \Sigma ; \Pi ; \\
& \Sigma^{i} ;\left(\left(\Pi^{i} ; \boldsymbol{\epsilon}^{(i)}\right),(\Pi ;), \ldots,(\Pi ;)^{(i)}\right) \\
& \Sigma ;((\Pi ; \alpha),(\Pi ; \alpha), \ldots,(\Pi ; \alpha)) \\
& \frac{\beta}{\alpha \rightarrow \beta}(\rightarrow \mathrm{I})^{(i)} \mapsto
\end{aligned}
$$

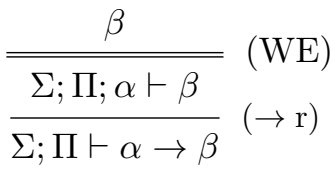

where with $(\Pi ; \alpha),(\Pi ; \alpha), \ldots,(\Pi ; \alpha)$ we denote an extensional multiset where the multiset $\Pi ; \alpha$ occurs at least twice and where $\Pi$ may be empty. Double line next to (WE) denotes possible several extensional contractions (at least one).

If the last rule is $(\rightarrow \mathrm{E})$, then we have the following cases:

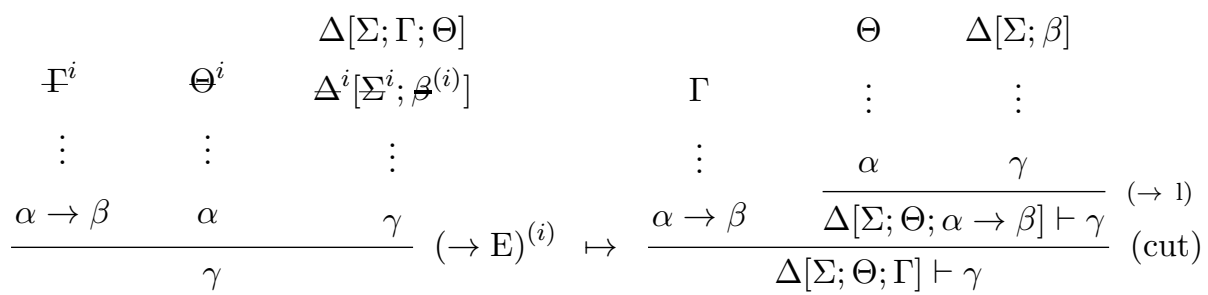

where

- $($ cut $)=($ cut $-i)$, if $\Gamma$ is non-empty,

- $($ cut $)=($ cut-ii $)$, if $\Gamma$ is empty and at least one of $\Theta$ or $\Sigma$ is non-empty, 
- $($ cut $)=($ cut - iii $)$, if $\Gamma$ and $\Theta$ are empty and $\Delta[\Sigma ; \beta]=\beta$.

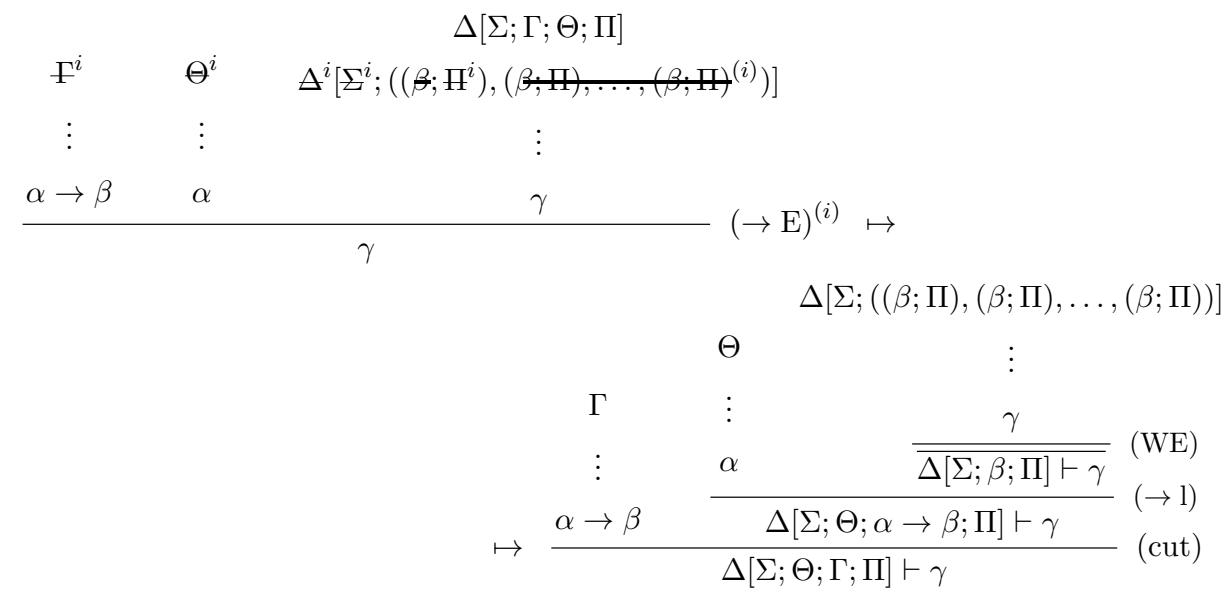

where

- $($ cut $)=($ cut $-\mathrm{i})$, if $\Gamma$ is non-empty,

- $($ cut $)=($ cut-ii $)$, if $\Gamma$ is empty and at least one of $\Theta, \Sigma$ or $\Pi$ is nonempty,

- $($ cut $)=($ cut-iii), if $\Gamma$ and $\Theta$ are empty and

$$
\Delta[\Sigma ;((\beta ; \Pi),(\beta ; \Pi), \ldots,(\beta ; \Pi))]=\beta, \beta, \ldots, \beta .
$$

If the last rule is ( $\circ \mathrm{I})$, then we have the following cases. If $\Sigma$ is nonempty, then:

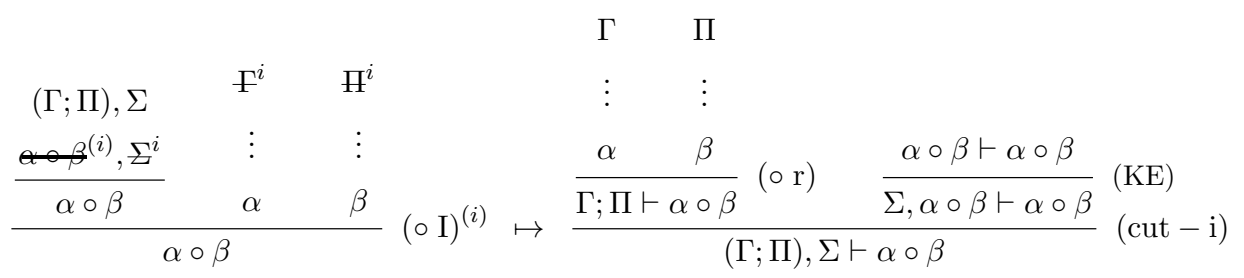

By the definition of the rule (o I), when $\Sigma$ is non-empty, then at least one of $\Gamma$ or $\Pi$ is non-empty, therefore the application of the rule (cut-i) is 
correct. Otherwise, we have:

$\begin{array}{ccccc}\Gamma ; \Pi & \Gamma^{i} & \Pi^{i} & \Gamma & \Pi \\ \frac{\beta^{(i)}}{\alpha \circ \beta} & \vdots & \vdots & \beta\end{array}(\circ \mathrm{I})^{(i)} \mapsto \frac{\alpha}{\Gamma ; \Pi \vdash \alpha \circ \beta}(\circ \mathrm{r})$

If the last rule is ( $\circ \mathrm{E})$, then we have the following cases:

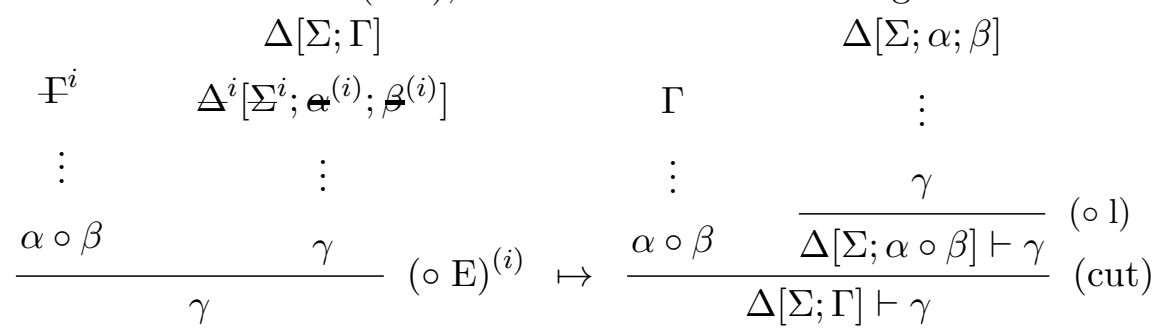

where (cut) is one of (cut-i), (cut-ii) or (cut-iii), depending on the multisets $\Gamma$ and $\Delta[\Sigma ; \alpha ; \beta]$, as above. Or:

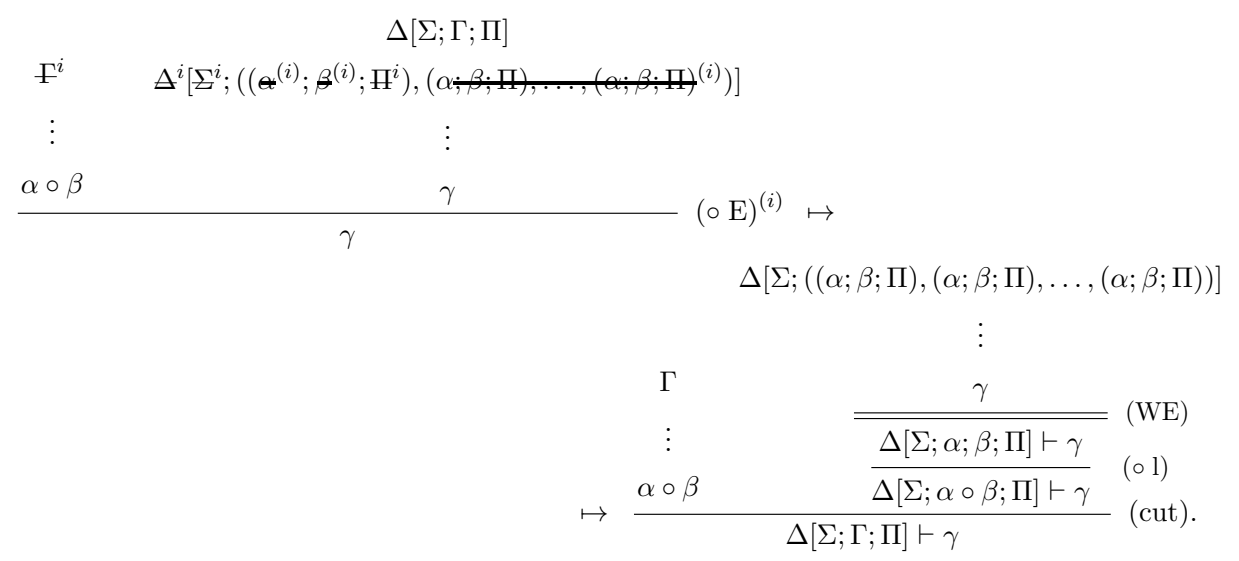


If the last rule is $(\wedge \mathrm{I})$, then we have:

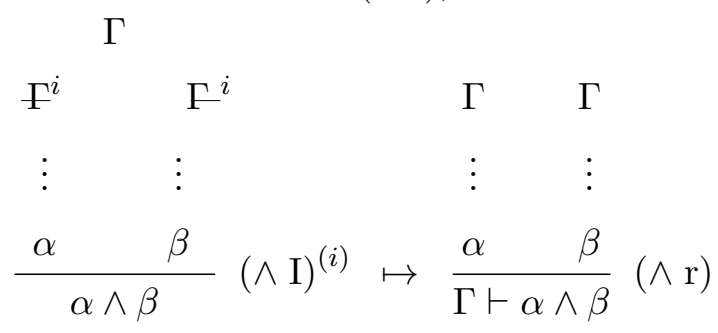

If the last rule is $(\wedge \mathrm{E})_{1}$, then we have the following cases:

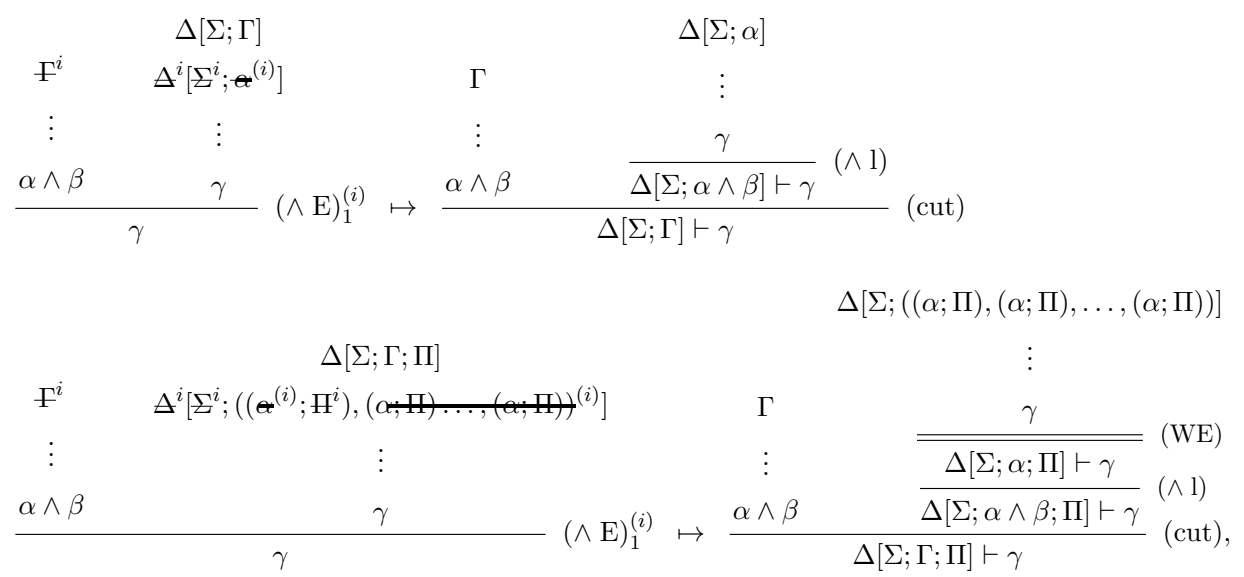

where (cut) is defined similarly as above. We can proceed similarly when the last rule is $(\wedge \mathrm{E})_{2}$.

If the last rule is $(\vee \mathrm{I})$, then we have:

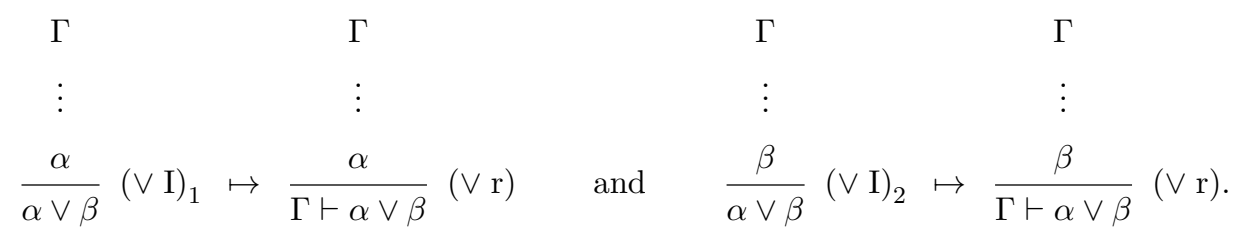


If the last rule is $(\vee \mathrm{E})$, then we have the following cases:
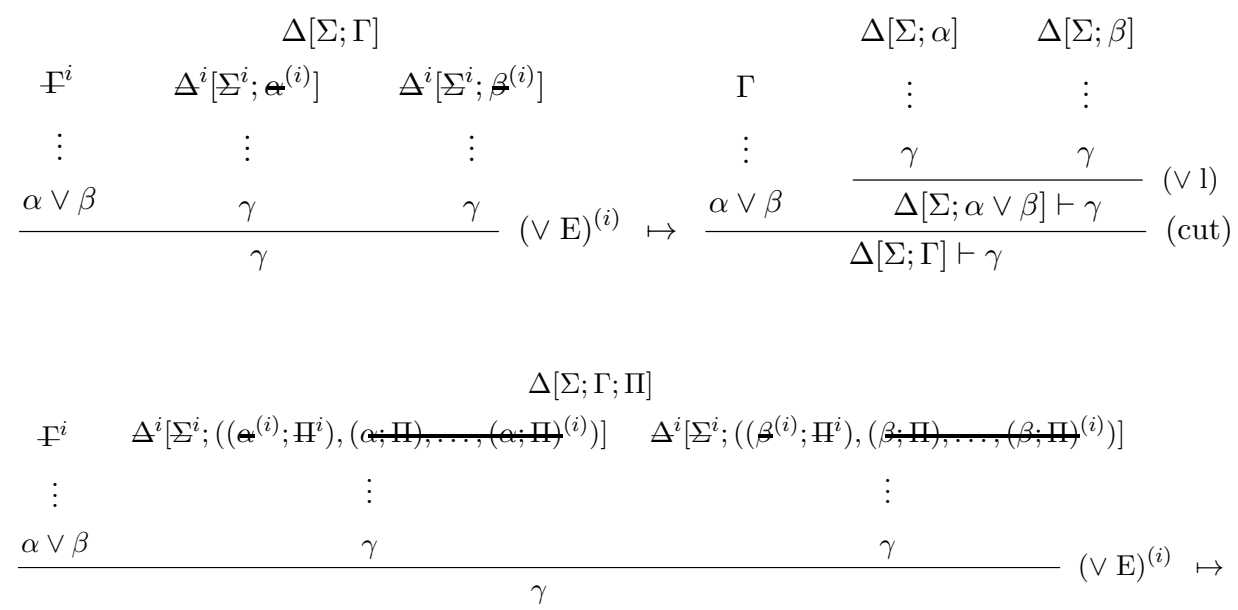

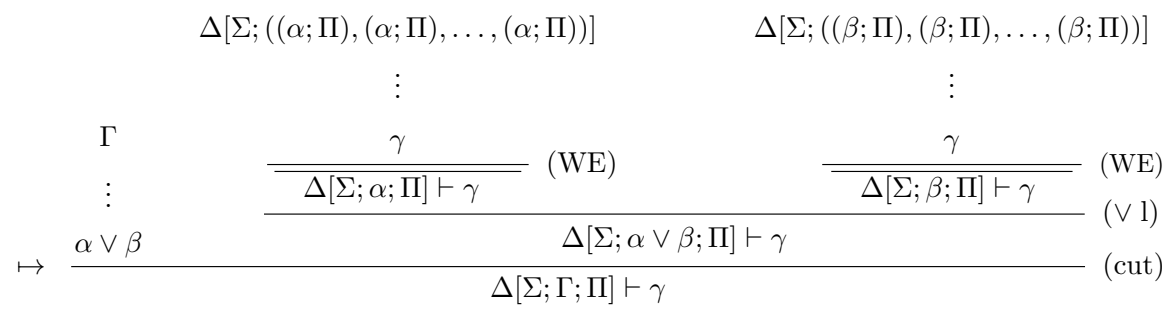

where (cut) is as above.

If the last rule is an initial rule, then we have:

$\frac{\alpha, \Gamma}{\alpha} \mapsto \frac{\alpha \vdash \alpha}{\alpha, \Gamma \vdash \alpha}(\mathrm{KE})$.

By the translation defined we have:

Theorem 2. If $d$ is an $N R W_{+}^{\circ}$-derivation of $\gamma$ from the multiset of open assumptions $\Gamma$, then its translation is a $G R W_{+}^{\circ}$-derivation of $\Gamma \vdash \gamma$.

$G R W_{+}^{\circ}$ and $N R W_{+}^{\circ}$ are equivalent systems meaning that:

Theorem 3. For any formula $\gamma$, the sequent $\vdash \gamma$ is provable in $G R W_{+}^{\circ}$ iff $\gamma$ is derivable from the empty multiset of open assumptions in $N R W_{+}^{\circ}$. 
Proof. From left to right, by Theorem 1., from right to left by Theorem 2.

Now we prove the following theorem:

Normalization Theorem. For every $N R W_{+}^{\circ}$-derivation of $\gamma$ from the empty multiset of open assumptions, there is a normal $N R W_{+}^{\circ}$-derivation of $\gamma$ from the empty multiset of open assumptions.

Proof. Let $d$ be a $N R W_{+}^{\circ}$-derivation of $\gamma$ from the empty multiset of open assumptions. Then by the translation defined, we obtain a $G R W_{+}^{\circ}-$ proof $\pi$ of $\vdash \gamma$. We eliminate cut in $\pi$, by the procedure given in [10] and then we transform that proof into the cut-free $\mathrm{KE}$-normal $G R W_{+}^{\circ}$ proof $\pi^{\prime}$. Finally, by the translation defined, we transform $\pi^{\prime}$ into the normal $N R W_{+}^{\circ}$-derivation of $\gamma$ from the empty multiset of open assumptions.

Corollary. In a normal derivation of $\gamma$ from the empty multiset of open assumptions, each formula in the derivation is a subformula of $\gamma$.

\section{Appendix}

We give the proof of the formula $(\alpha \rightarrow(\beta \wedge \delta)) \wedge((\gamma \wedge \delta) \rightarrow \gamma) . \rightarrow .((\alpha \vee$ $\gamma) \wedge(\alpha \vee \delta)) \rightarrow(\beta \vee \gamma)$ in Brady's, Dunn's and in our system.

Dunn's proof:

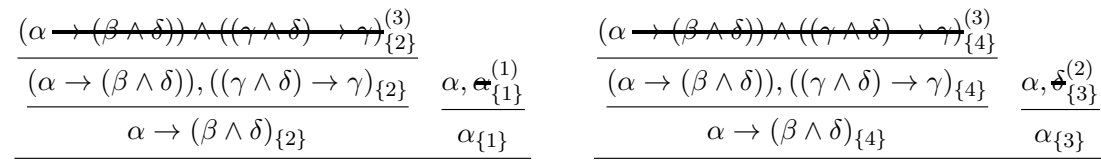

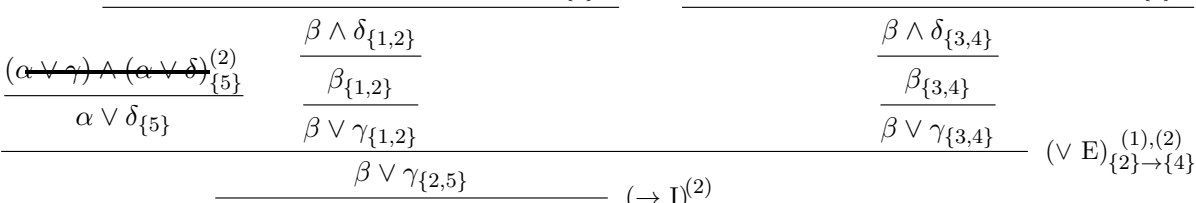

$$
\begin{aligned}
& \frac{\frac{}{((\alpha \vee \gamma) \wedge(\alpha \vee \delta)) \rightarrow(\beta \vee \gamma)_{\{2\}}}(\rightarrow \mathrm{I})^{(2)}}{(\alpha \rightarrow(\beta \wedge \delta)) \wedge((\gamma \wedge \delta) \rightarrow \gamma) . \rightarrow .((\alpha \vee \gamma) \wedge(\alpha \vee \delta)) \rightarrow(\beta \vee \gamma)_{\{\}}}(\rightarrow \mathrm{I})^{(3)}
\end{aligned}
$$


In this proof, at least one of the formulae from the assumption set $\alpha, \alpha$ is used (i.e. the crossed out formula $\alpha$ ) and at least one of the formulae from the assumption set $\alpha, \delta$ is used (i.e. the crossed out formula $\delta$ ). Those formulae are discharged by the application of the disjunction elimination rule. This is a context-sharing rule, meaning that the derivations of its premises $\beta \vee \gamma_{\{1,2\}}$ and $\beta \vee \gamma_{\{3,4\}}$, should share the same assumptions; thus the assumptions $(\alpha \rightarrow(\beta \wedge \delta)) \wedge((\gamma \wedge \delta) \rightarrow \gamma)_{\{2\}}$ and $(\alpha \rightarrow(\beta \wedge \delta)) \wedge((\gamma \wedge \delta) \rightarrow$ $\gamma)_{\{4\}}$ must be equal. To achieve that in practice, we reassign $\{4\}$ with $\{2\}$.

Brady's proof (as it is given in [3]):

\begin{tabular}{|c|c|c|}
\hline 1 & $(\alpha \rightarrow(\beta \wedge \delta)) \wedge((\gamma \wedge \delta) \rightarrow \gamma)_{\{1\}}$ & \\
\hline 2 & $\alpha \rightarrow(\beta \wedge \delta)_{\{1\}}$ & $\wedge \mathrm{E}, 1$ \\
\hline 3 & $(\gamma \wedge \delta) \rightarrow \gamma_{\{1\}}$ & $\wedge \mathrm{E}, 1$ \\
\hline 4 & $(\alpha \vee \gamma) \wedge(\alpha \vee \delta)_{\{2\}}$ & \\
\hline 5 & $\alpha \vee \gamma_{\{2\}}$ & $\wedge \mathrm{E}, 4$ \\
\hline 5 & $\alpha \vee \delta_{\{2\}}$ & $\wedge \mathrm{E}, 4$ \\
\hline 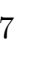 & $\alpha, \gamma_{\{2\}}$ & $\mathrm{VE}, 5$ \\
\hline 8 & $\alpha, \delta_{\{2\}}$ & $\vee \mathrm{E}, 6$ \\
\hline 9 & $\alpha, \gamma \vee \delta_{\{2\}}$ & $\vee \mathrm{I}, 7$ \\
\hline 10 & $\beta \wedge \delta, \gamma_{\{1,2\}}$ & $\rightarrow \mathrm{E}, 2,7$ \\
\hline 11 & $\beta, \gamma_{\{1,2\}}$ & $\wedge \mathrm{E}, 10$ \\
\hline 12 & $\beta \vee \gamma, \gamma_{\{1,2\}}$ & $\vee \mathrm{I}, 11$ \\
\hline 13 & $\beta \vee \gamma, \beta \vee \gamma_{\{1,2\}}$ & VI, 12 \\
\hline 14 & $\beta \vee \gamma_{\{1,2\}}$ & ,E, 13 \\
\hline 15 & $(\alpha \vee \gamma) \wedge(\alpha \vee \delta) \rightarrow(\beta \vee \gamma)_{\{1\}}$ & $\rightarrow \mathrm{I}, 4,14$ \\
\hline & $(\alpha \rightarrow(\beta \wedge \delta)) \wedge((\gamma \wedge \delta) \rightarrow \gamma) . \rightarrow .(\alpha \vee \gamma) \wedge(\alpha \vee \delta) \rightarrow(\beta \vee \gamma)_{\{\}}$ & $\rightarrow \mathrm{I}, 1,16$ \\
\hline
\end{tabular}

We now describe our proof of the same formula. We begin our derivation with the initial rules: $\frac{\alpha \rightarrow(\beta \wedge \delta)}{\alpha \rightarrow(\beta \wedge \delta)}, \frac{\gamma, \alpha}{\alpha}$ and $\frac{\beta \wedge \gamma}{\beta}$. The initial rule $\frac{\gamma, \alpha}{\alpha}$ means that from $\gamma, \alpha$ we can derive $\alpha$. This is the correct derivation, since $\gamma, \alpha$ stands in for $\gamma \wedge \alpha$ and from $\gamma \wedge \alpha$ we can derive $\alpha$ in our logic. Then we apply the rule ( $\rightarrow \mathrm{E}$ ) (see below). We derive $\beta$ and discharge the 
assumption $\beta \wedge \delta$, taking $\alpha \rightarrow(\beta \wedge \delta)$ together with $\gamma, \alpha$ instead; namely, in lieu of $\beta \wedge \delta$ we place the intensional multiset $\alpha \rightarrow(\beta \wedge \delta) ;(\gamma, \alpha)$, and we write it above the discharged assumption $\beta \wedge \delta$. The discharged assumption $\beta \wedge \delta$ is crossed out by the thicker line than $\alpha \rightarrow(\beta \wedge \delta)$ and $\gamma, \alpha-$ the later assumptions are still open, however not at the starting position. The multiset $\alpha \rightarrow(\beta \wedge \delta) ;(\gamma, \alpha)$ is the multiset of moved open assumptions. The derivation:

$$
\frac{\frac{\alpha \rightarrow(\beta \wedge \delta)^{1}}{\alpha \rightarrow(\beta \wedge \delta)}{\frac{\gamma, \alpha^{1}}{\alpha}}^{\alpha \rightarrow(\beta \wedge \delta) ;(\gamma, \alpha)}}{\beta}(\rightarrow \mathrm{E})^{(1)}
$$

is the derivation of $\beta$ from the multiset of open assumptions $\alpha \rightarrow(\beta \wedge$ $\delta) ;(\gamma, \alpha)$ (this multiset has the same informal meaneing as the formula $(\alpha \rightarrow(\beta \wedge \delta)) \circ(\gamma \wedge \alpha)$ from which we can derive $\beta)$. Then we apply the rule $(\vee \mathrm{I})$ and after that, the rule $(\wedge \mathrm{E})$ :

$(*)$

$$
\begin{aligned}
& (\alpha \rightarrow(\beta \wedge \delta)) \wedge((\gamma \wedge \delta) \rightarrow \gamma) ;(\gamma, \alpha) \\
& \alpha \longrightarrow(\beta \wedge \delta)^{(2)} ;(\gamma, \alpha)^{2}
\end{aligned}
$$

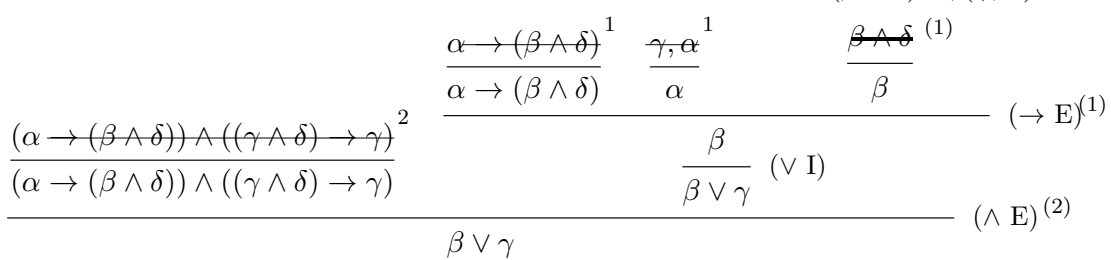

By the application of the rule $(\wedge \mathrm{E})$ the formula $\alpha \rightarrow(\beta \wedge \delta)$ from the assumption multiset $\alpha \rightarrow(\beta \wedge \delta) ;(\gamma, \alpha)$ has been discharged and substituted by the formula $\alpha \rightarrow(\beta \wedge \delta)) \wedge((\gamma \wedge \delta) \rightarrow \gamma$. The new assumption multiset is now $(\alpha \rightarrow(\beta \wedge \delta)) \wedge((\gamma \wedge \delta) \rightarrow \gamma) ;(\gamma, \alpha)$ and it is written above $\alpha \rightarrow$ $(\beta \wedge \delta) ;(\gamma, \alpha)$. We shall denote the above derivation as:

$$
\begin{aligned}
(\alpha \rightarrow(\beta \wedge \delta)) \wedge & ((\gamma \wedge \delta) \rightarrow \gamma) ;(\gamma, \alpha) \\
\operatorname{der}_{1}: \quad & D_{1} \\
\beta & \vee \gamma
\end{aligned}
$$


The formulae $\alpha \rightarrow(\beta \wedge \delta)^{1}$ and $\alpha \rightarrow(\beta \wedge \delta)^{(2)}$ are one and the same. The former one indicates that the formula $\alpha \rightarrow(\beta \wedge \delta)$ is moved by the application of the rule $(\rightarrow \mathrm{E})^{(1)}$ and the later one indicates that it is discharged by the application of the rule $(\wedge \mathrm{E})^{(2)}$. Given that, the above derivation could also be presented in the following form:

$$
\frac{\frac{(\alpha \rightarrow(\beta \wedge \delta)) \wedge((\gamma \wedge \delta) \rightarrow \gamma)^{2}}{(\alpha \rightarrow(\beta \wedge \delta)) \wedge((\gamma \wedge \delta) \rightarrow \gamma)}}{\beta \vee \gamma}(\rightarrow \mathrm{E})^{(1)}
$$

(Note the double superscript $\underset{(2)}{1}$ in $\alpha \rightarrow(\beta \wedge \delta)^{(2)}$.)

Remember that our translations from sequent calculus to natural deduction have this form, i.e. they are without explicitly given multisets of moved open assumptions. However, those multisets can be easily restored, as follows. Given the above derivation, we first generate an intensional multiset of all assumption multisets with moved label 1, i.e. we generate the multiset $\alpha \rightarrow(\beta \wedge \delta) ;(\gamma, \alpha)$, which we then place in lieu of the formula with the discharge label (1). i.e. in lieu of the formula $\beta \_\delta^{(1)}$ :

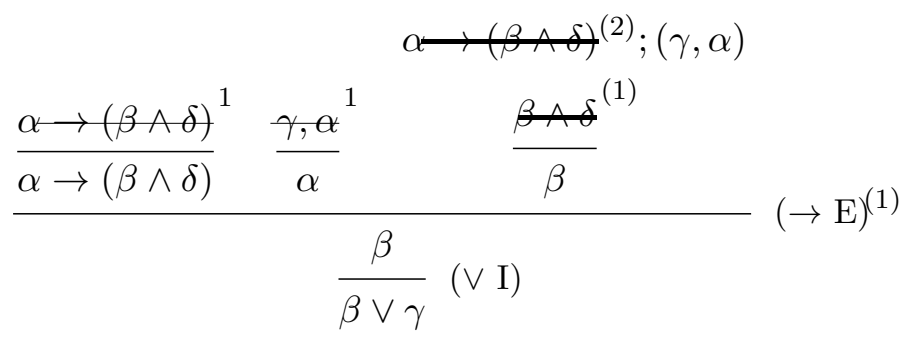

Now, by the application of the rule $(\wedge \mathrm{E})$, we substitute the formula $\propto)^{(2)}$ (whose discharge label $\left.(2)\right)$ with the formula $(\alpha \rightarrow(\beta \wedge \delta)) \wedge$ $((\gamma \wedge \delta) \rightarrow \gamma)$ (whose moved label is 2$)$ and we obtain the derivation $(*)$ above.

We now go back to our derivation. Next we construct the derivation of the formula $\beta \vee \gamma$ from the multiset of open assumptions $(\alpha \rightarrow(\beta \wedge \delta) \wedge$ $((\gamma \wedge \delta) \rightarrow \gamma) ;(\gamma, \delta):$ 


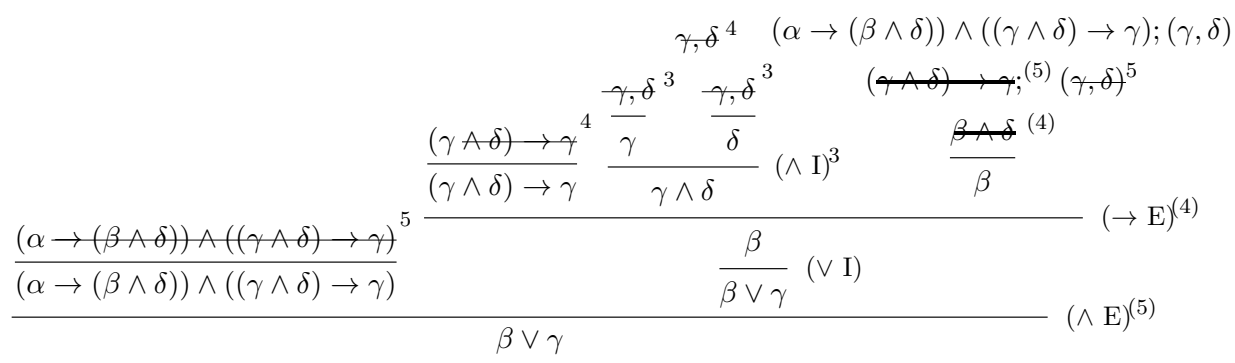

First we apply the rule $(\wedge \mathrm{I})$. This is a context-sharing rule meaning that the derivation of its premises $\gamma$ and $\delta$ must share the same assumptions. Therefore $\gamma, \delta$ in $\frac{\gamma, \delta}{\gamma}$ and $\gamma, \delta$ in $\frac{\gamma, \delta}{\delta}$ must be equal. To obtain that, instead of these two multisets, we take a single multiset $\gamma, \delta$. This is denoted by crossing out multisets $\gamma, \delta$ and $\gamma, \delta$ and writing a single multiset $\gamma, \delta$ above them. (Sara Negri in her natural deduction calculus for linear logic [15] uses assumption labels and she takes the control over the assumptions in context-sharing rules by assigning the same label to those assumptions, treating formulae with the same label as if they were the same formulae. Using assumption labels in our natural deduction calculus would be very complicated due to the presence of two types of multisets of formula, extensional and intensional ones, which must be allowed to be nested within one another.)

We shall denote the above derivation as:

$$
\begin{aligned}
(\alpha \rightarrow(\beta \wedge \delta)) \wedge & ((\gamma \wedge \delta) \rightarrow \gamma) ;(\gamma, \delta) \\
\operatorname{der}_{2}: \quad & D_{2} \\
\beta & \vee \gamma
\end{aligned}
$$

With $\operatorname{der}_{1}$ and $d e r_{2}$ we construct the derivation of the formula $\beta \vee \gamma$ from the multiset of open assumptions $(\alpha \rightarrow(\beta \wedge \delta)) \wedge((\gamma \wedge \delta) \rightarrow \gamma) ;(\gamma, \alpha \vee \delta)$ :

$$
\begin{aligned}
& (\alpha \rightarrow(\beta \wedge \delta)) \wedge((\gamma \wedge \delta) \rightarrow \gamma) ;(\gamma, \alpha \vee \delta) \\
& (\alpha \rightarrow(\beta \wedge \delta)) \wedge((\gamma \wedge \delta) \rightarrow \gamma)^{6} ;\left(\gamma^{6}, \boldsymbol{e}^{(6)}\right) \quad(\alpha \rightarrow(\beta \wedge \delta)) \wedge((\gamma \wedge \delta) \rightarrow \gamma)^{6} ;\left(\gamma^{6}, \boldsymbol{\mathcal { f }}^{(6)}\right)
\end{aligned}
$$

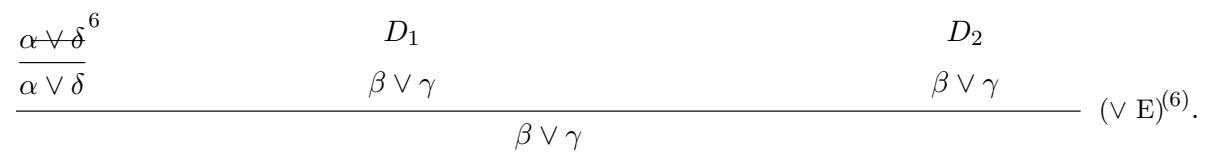


By the application of the rule $(\vee \mathrm{E})$, we discharge the formula $\alpha$ from the multiset of open assumptions $(\alpha \rightarrow(\beta \wedge \delta)) \wedge((\gamma \wedge \delta) \rightarrow \gamma) ;(\gamma, \boldsymbol{\theta})$ (the discharged formula is crossed out) and also the formula $\delta$ from the multiset $(\alpha \rightarrow(\beta \wedge \delta)) \wedge((\gamma \wedge \delta) \rightarrow \gamma) ;(\gamma, \delta)$ and take the formula $\alpha \vee \delta$ instead of them. The assumption multisets of the derivations $D_{1}$ and $D_{2}$ are now equal. The rule $(\mathrm{V} \mathrm{E})$ is a context-sharing rule, meaning that the derivation $D_{1}$ and $D_{2}$ should share the same assumptions. For this reason, instead of two equal assumption multisets, we take a single multiset of moved open assumptions of the form $(\alpha \rightarrow(\beta \wedge \delta)) \wedge((\gamma \wedge \delta) \rightarrow \gamma) ;(\gamma, \alpha \vee \delta)$ and we write it above them.

We shall denote the above derivation as:

$$
\begin{gathered}
(\alpha \rightarrow(\beta \wedge \delta)) \wedge((\gamma \wedge \delta) \rightarrow \gamma) ;(\gamma, \alpha \vee \delta) \\
D_{3} \\
\beta \vee \gamma
\end{gathered}
$$

Similarly we construct the derivation of $\beta \vee \gamma$ from the multiset of open assumptions $(\alpha \rightarrow(\beta \wedge \delta)) \wedge((\gamma \wedge \delta) \rightarrow \gamma) ;(\alpha, \alpha \vee \delta)$ :

$$
\begin{aligned}
& (\alpha \rightarrow(\beta \wedge \delta)) \wedge((\gamma \wedge \delta) \rightarrow \gamma) ;(\alpha, \alpha \vee \delta) \\
& \alpha \longmapsto(\beta \wedge \delta)^{(8)} ;(\alpha, \alpha \vee \delta)^{8} \\
& \frac{\frac{(\alpha \rightarrow(\beta \wedge \delta)) \wedge((\gamma \wedge \delta) \rightarrow \gamma)^{8}}{(\alpha \rightarrow(\beta \wedge \delta)) \wedge((\gamma \wedge \delta) \rightarrow \gamma)}}{\beta \vee(\beta \wedge \delta)} \frac{\frac{\alpha \rightarrow(\beta \wedge \delta)^{7}}{\alpha \rightarrow(7)}}{\frac{\alpha, \alpha \vee \delta^{7}}{\alpha}}(\rightarrow \mathrm{E})^{(7)}
\end{aligned}
$$

We shall denote the above derivation as:

$$
\begin{gathered}
(\alpha \rightarrow(\beta \wedge \delta)) \wedge((\gamma \wedge \delta) \rightarrow \gamma) ;(\alpha, \alpha \vee \delta) \\
D_{4} \\
\beta \vee \gamma
\end{gathered}
$$

We now give the whole derivation of the formula $(\alpha \rightarrow(\beta \wedge \delta)) \wedge((\gamma \wedge$ $\delta) \rightarrow \gamma) . \rightarrow .((\alpha \vee \gamma) \wedge(\alpha \vee \delta)) \rightarrow(\beta \vee \gamma)$ in our natural deduction calculus: 


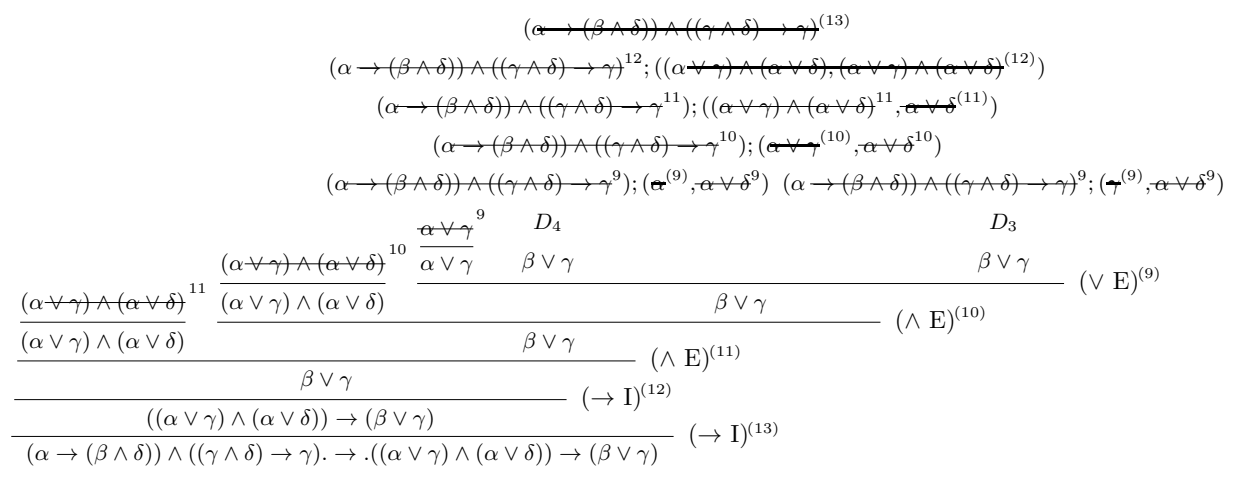

Our proof is neither the shortest one nor the simplest one, however it is the only one which is without relevance numerals, and the only one which admits the simple translation into a sequent calculus proof and vice versa, as we have seen above.

\section{Acknowledgments}

I would like to thank the reviewer for the very helpful comments and recommendations. This work is supported by the Ministry of Science and Technology of Serbia, grant number ON174026.

\section{References}

[1] A. Anderson and N. Belnap Jr., Entailment: the logic of relevance and necessity, vol. 1, Princeton University Press, Princeton, New Jersey, 1975.

[2] K. Bimbo, $L E_{\rightarrow}^{t}, L R_{\wedge \sim}^{\circ}, L K$ and cutfree proofs, Journal of Philosophical Logic 36 (2007), 557-570.

[3] R. T. Brady, Normalized natural deduction system for some relevant logics I: The logic DW, Journal of Symbolic Logic 7:1 (2006), 35-66.

[4] A. Church, The weak theory of implication, Kontrolliertes Denken (Festgabe zum 60. Geburtstag von Prof. W. Britzelmayr), Munich 1951.

[5] J.M. Dunn, A 'Gentzen system' for positive relevant implication, The Journal of Symbolic Logic 38 (1973), 356-357.

[6] J.M. Dunn, Relevance logic and entailment, Handbook of Philosophical Logic, vol. 3, D. Gabbay and F. Guenthner (eds.), D. Reidel Publishing Company, pp. 117-224, 1986. 
[7] J.M. Dunn and G. Restall, Relevance logic, Handbook of Philosophical Logic, vol. 6, D. Gabbay and F. Guenthner (eds.), Kluwer Academic Publlishers, pp. 1-128, 2002 .

[8] G. Gentzen, Collected Papers, (ed. M. E. Szabo), North-Holland, Amsterdam, 1969.

[9] S. Giambrone, $T W_{+}$and $R W_{+}$are decidable, Journal of Philosophical Logic 14 (1985), 235-254.

[10] M. Ilić, An alternative Gentzenization of $R W_{+}^{\circ}$, Mathematical Logic Quarterly 62:6 (2016), 465-480.

[11] A. Kron, Decidability and interpolation for a first-order relevance logic, Substructural Logics, P. Schroeder-Heister and K. Došen (eds.), Oxford University Press, pp. 153-177, 1993.

[12] R.K. Meyer and M.A. McRobbie, Multisets and relevant implication I and II, Australian Journal of Philosophy 60:2 (1982), 107-139, and 3 (1982), 265-281.

[13] G. Minc, Cut elimination theorem for relevant logics, Journal of Soviet Mathematics 6 (1976), 422-428.

[14] S. Negri and J. von Plato, Structural Proof Theory, Cambridge University Press, 2001.

[15] S. Negri, A normalizing system of natural deduction for intuitionistic linear logic, Archive for Mathematical Logic 41 (2002), 789-810.

[16] J. von Plato, Natural deduction with general elimination rules, Archive for Mathematical Logic 40 (2001), 541-567.

[17] D. Prawitz, Natural Deduction. A Proof-theoretical Study, Almquist and Wiksell, Stockholm, 1965.

[18] A. Urquhart, Relevance logic: problems open and closed, Australian Journal of Logic 13:1 (2016), Article no. 2.

Faculty of Economics

University of Belgrade

Kamenička 6, 11000 Belgrade, Serbia

mirjanailic@ekof.bg.ac.rs 